TRANSACTIONS OF THE

AMERICAN MATHEMATICAL SOCIETY

Volume 365, Number 7, July 2013, Pages 3493-3534

S 0002-9947(2013)05716-9

Article electronically published on February 21, 2013

\title{
GAUSSIAN SUBORDINATION FOR THE BEURLING-SELBERG EXTREMAL PROBLEM
}

\author{
EMANUEL CARNEIRO, FRIEDRICH LITTMANN, AND JEFFREY D. VAALER
}

\begin{abstract}
We determine extremal entire functions for the problem of majorizing, minorizing, and approximating the Gaussian function $e^{-\pi \lambda x^{2}}$ by entire functions of exponential type. This leads to the solution of analogous extremal problems for a wide class of even functions that includes most of the previously known examples, plus a variety of new interesting functions such as $|x|^{\alpha}$ for $-1<\alpha$; $\log \left(\left(x^{2}+\alpha^{2}\right) /\left(x^{2}+\beta^{2}\right)\right)$, for $0 \leq \alpha<\beta ; \log \left(x^{2}+\alpha^{2}\right)$; and $x^{2 n} \log x^{2}$, for $n \in \mathbb{N}$. Further applications to number theory include optimal approximations of theta functions by trigonometric polynomials and optimal bounds for certain Hilbert-type inequalities related to the discrete Hardy-Littlewood-Sobolev inequality in dimension one.
\end{abstract}

\section{INTRODUCTION}

We recall that an entire function $F: \mathbb{C} \rightarrow \mathbb{C}$ is of exponential type at most $2 \pi \delta$ if for every $\epsilon>0$ there exists a positive constant $C$, such that the inequality

$$
|F(z)| \leq C e^{(2 \pi \delta+\epsilon)|z|}
$$

holds for all complex numbers $z$. We say that $F: \mathbb{C} \rightarrow \mathbb{C}$ is a real entire function if the restriction of $F$ to $\mathbb{R}$ is real valued. For a given function $f: \mathbb{R} \rightarrow \mathbb{R}$, the Beurling-Selberg minorization problem consists of finding a real entire function $S(z)$ of exponential type at most $2 \pi \delta$, such that $S(x) \leq f(x)$ for all real $x$, and the integral

$$
\int_{-\infty}^{\infty}\{f(x)-S(x)\} \mathrm{d} x
$$

is minimized. In the Beurling-Selberg majorization problem we seek a real entire function $T(z)$ of exponential type at most $2 \pi \delta$, such that $f(x) \leq T(x)$ for all real $x$, and the integral

$$
\int_{-\infty}^{\infty}\{T(x)-f(x)\} \mathrm{d} x
$$

is minimized. These extremal problems were solved in unpublished work of A. Beurling in the late 1930s for the function $f(x)=\operatorname{sgn}(x)$. Later A. Selberg used translates of Beurling's extremal function to majorize and minorize the characteristic function of an interval. Selberg made use of this construction to obtain a sharp form of the large sieve inequality. A more detailed account of the history of these discoveries,

Received by the editors February 1, 2010 and, in revised form, July 12, 2011.

2010 Mathematics Subject Classification. Primary 41A30, 41A52; Secondary 41A05, 41A44, $42 \mathrm{~A} 82$.

Key words and phrases. Gaussian, exponential type, extremal functions, majorization, tempered distributions. 
together with further applications, is given in [28] and [29]. A further discussion of the early development of this theory, including simple proofs of the Erdös-Turán inequality and the Montgomery-Vaughan inequality (see [25]), is presented in [35].

General solutions to the Beurling-Selberg extremal problem for different classes of functions have been identified in several recent papers, and these have included new number theoretic applications. A key ingredient in most applications is the close connection between entire functions of exponential type and functions with compactly supported Fourier transform via the Paley-Wiener theorem. The extremal problem for the exponential function $f(x)=e^{-\lambda|x|}, \lambda>0$, is discussed by Graham and Vaaler in [14, with applications to Tauberian theorems. The problem for $f(x)=x^{n} \operatorname{sgn}(x)$ and $f(x)=\left(x^{+}\right)^{n}$, where $n$ is a positive integer, is considered by Littmann in [21, 22] and [23]. In [3] and 4, Carneiro and Vaaler extended the construction of extremal approximations for a class of even functions that includes $f(x)=\log |x|, f(x)=\log \left(x^{2} /\left(x^{2}+4\right)\right)$ and $f(x)=|x|^{\alpha}$, with $-1<\alpha<1$.

Recently, Chandee and Soundararajan in [6] used the extremal functions associated to $f(x)=\log \left(x^{2} /\left(x^{2}+4\right)\right)$ to obtain improved upper bounds for $\left|\zeta\left(\frac{1}{2}+i t\right)\right|$ assuming the Riemann Hypothesis $(\mathrm{RH})$. They remarked that the extremals for the function $f(x)=\log \left(\left(x^{2}+\alpha^{2}\right) /\left(x^{2}+4\right)\right)$, for $\alpha \neq 0$, not contemplated in the previous literature, naturally arise in bounding $\left|\zeta\left(\frac{1}{2} \pm \alpha+i t\right)\right|$, assuming $\mathrm{RH}$. We will return to this example later in this paper, since the family of functions $f(x)=\log \left(\left(x^{2}+\alpha^{2}\right) /\left(x^{2}+\beta^{2}\right)\right)$, for $0 \leq \alpha<\beta$, is included in the general results we obtain here. The corresponding bounds for $\left|\zeta\left(\frac{1}{2} \pm \alpha+i t\right)\right|$, assuming $\mathrm{RH}$, have been obtained by Chandee and Carneiro in [5].

For a given function $f: \mathbb{R} \rightarrow \mathbb{R}$, it is also instructive to consider the analogous approximation problem. In this case we seek an entire function $F(z)$ of exponential type at most $2 \pi \delta$, such that the integral

$$
\int_{-\infty}^{\infty}|f(x)-F(x)| \mathrm{d} x
$$

is minimized. The approximation problem has a much more extensive literature, as discussed in Ganzburg [9], 10], [11], Krein [19], and Sz.-Nagy [26]. We find that the solutions to the approximation problem are obtained by constructing an entire function that interpolates the values of $f(x)$ at an additive coset of $\mathbb{Z}$ in $\mathbb{R}$. The solutions to the majorization and minorization problems involve a similar construction that interpolates the values of $f(x)$ and the derivative of $f(x)$ at an additive coset of $\mathbb{Z}$ in $\mathbb{R}$.

In addition to the references already mentioned, other problems on approximation by entire functions and trigonometric polynomials have been investigated by Carneiro [2, Ganelius [8], Ganzburg and Lubinsky [12, Graham and Vaaler [15], Montgomery 24] and Vaaler [36. Related extremal problems in several variables are considered by Barton, Montgomery and Vaaler [1], Holt and Vaaler [17, and $\mathrm{Li}$ and Vaaler 20].

This paper is divided into three parts. In the first part we consider the problem of majorizing, minorizing, and approximating the Gaussian function

$$
x \mapsto G_{\lambda}(x)=e^{-\pi \lambda x^{2}}
$$

on $\mathbb{R}$ by entire functions of exponential type. Here $\lambda>0$ is a parameter. We make use of classical interpolation techniques and integral representations to achieve this goal. 
The second part is independent of the first, and presents a solution to the Beurling-Selberg extremal problem based on the theory of distributions. Such methods have been used before in [9, [10, 11], and [12], in work on the two-sided approximation problem. The main ingredient in this approach is the Paley-Wiener theorem for distributions. This leads to the solution of the extremal problem for a wide class of even functions once one knows the solution for a family of even functions with an independent parameter. In the present work the family of even function we use is given by (0.4) for $\lambda>0$.

Various applications are presented in the third part of the paper. Most of the previously known cases become corollaries of our distribution theorems (for instance, the results in [3], 4], 14] and [22]), and we obtain the solution to the extremal problems for new interesting functions such as $|x|^{\alpha}$ for $-1<\alpha$; $\log \left(\left(x^{2}+\alpha^{2}\right) /\left(x^{2}+\beta^{2}\right)\right)$, for $0 \leq \alpha<\beta$; $\log \left(x^{2}+\alpha^{2}\right)$; and $|x|^{2 n} \log |x|$, for $n \in \mathbb{N}$. Some of the extremal $L^{1}(\mathbb{R})$-approximations (without the one-sided condition) have previously been obtained by Sz.-Nagy (cf. [32, Chapter 7]). Further applications to number theory include optimal approximations of theta functions by trigonometric polynomials and optimal bounds for certain Hilbert-type inequalities related to the discrete Hardy-Littlewood-Sobolev inequality in dimension one.

\section{Part I: The Gaussian}

\section{The extremal problem for the Gaussian}

For each positive value of $\lambda$ we define three entire functions of the complex variable $z$ as follows:

$$
\begin{aligned}
& K_{\lambda}(z)=\left(\frac{\cos \pi z}{\pi}\right)\left\{\sum_{n=-\infty}^{\infty}(-1)^{n+1} \frac{G_{\lambda}\left(n+\frac{1}{2}\right)}{\left(z-n-\frac{1}{2}\right)}\right\}, \\
& L_{\lambda}(z)=\left(\frac{\cos \pi z}{\pi}\right)^{2}\left\{\sum_{m=-\infty}^{\infty} \frac{G_{\lambda}\left(m+\frac{1}{2}\right)}{\left(z-m-\frac{1}{2}\right)^{2}}+\sum_{n=-\infty}^{\infty} \frac{G_{\lambda}^{\prime}\left(n+\frac{1}{2}\right)}{\left(z-n-\frac{1}{2}\right)}\right\}, \\
& M_{\lambda}(z)=\left(\frac{\sin \pi z}{\pi}\right)^{2}\left\{\sum_{m=-\infty}^{\infty} \frac{G_{\lambda}(m)}{(z-m)^{2}}+\sum_{n=-\infty}^{\infty} \frac{G_{\lambda}^{\prime}(n)}{(z-n)}\right\} .
\end{aligned}
$$

The function $K_{\lambda}(z)$ is an entire function of exponential type $\pi$ which interpolates the values of the function $G_{\lambda}(z)$ at points of the coset $\mathbb{Z}+\frac{1}{2}$. We will show that among all entire functions of exponential type at most $\pi$, the function $K_{\lambda}(z)$ provides the best approximation to $G_{\lambda}(z)$ with respect to the $L^{1}$-norm on $\mathbb{R}$.

The function $L_{\lambda}(z)$ is a real entire function of exponential type $2 \pi$ which interpolates both the values of $G_{\lambda}(z)$ and the values of its derivative $G_{\lambda}^{\prime}(z)$ on the coset $\mathbb{Z}+\frac{1}{2}$. Similarly, the function $M_{\lambda}(z)$ is a real entire function of exponential type $2 \pi$ which interpolates both the values of $G_{\lambda}(z)$ and the values of its derivative $G_{\lambda}^{\prime}(z)$ on the integers $\mathbb{Z}$. We will show that these functions satisfy the basic inequality

$$
L_{\lambda}(x) \leq G_{\lambda}(x) \leq M_{\lambda}(x)
$$

for all real $x$. Moreover, we will show that the value of each of the two integrals

$$
\int_{-\infty}^{\infty}\left\{G_{\lambda}(x)-L_{\lambda}(x)\right\} \mathrm{d} x \text { and } \int_{-\infty}^{\infty}\left\{M_{\lambda}(x)-G_{\lambda}(x)\right\} \mathrm{d} x,
$$

is minimized. 
In order to state a more precise form of our main results for the Gaussian function, we make use of the basic theta functions. Here $v$ is a complex variable, $\tau$ is a complex variable with $\Im\{\tau\}>0, q=e^{\pi i \tau}$, and $e(z)=e^{2 \pi i z}$. Our notation for the theta functions is standard and follows that of Chandrasekharan [7. Thus we define

$$
\begin{aligned}
& \theta_{1}(v, \tau)=\sum_{n=-\infty}^{\infty} q^{\left(n+\frac{1}{2}\right)^{2}} e\left(\left(n+\frac{1}{2}\right) v\right), \\
& \theta_{2}(v, \tau)=\sum_{n=-\infty}^{\infty}(-1)^{n} q^{n^{2}} e(n v), \\
& \theta_{3}(v, \tau)=\sum_{n=-\infty}^{\infty} q^{n^{2}} e(n v) .
\end{aligned}
$$

We note that for a fixed value of $\tau$ with $\Im\{\tau\}>0$, each of the functions $v \mapsto \theta_{1}(v, \tau)$, $v \mapsto \theta_{2}(v, \tau)$, and $v \mapsto \theta_{3}(v, \tau)$ is an even entire function of $v$. The function $v \mapsto \theta_{1}(v, \tau)$ is periodic with period 2 , and satisfies the identity

$$
\theta_{1}(v+1, \tau)=-\theta_{1}(v, \tau)
$$

for all complex $v$. Both of the functions $v \mapsto \theta_{2}(v, \tau)$, and $v \mapsto \theta_{3}(v, \tau)$, are periodic with period 1 . They are related by the identity

$$
\theta_{2}\left(v+\frac{1}{2}, \tau\right)=\theta_{3}(v, \tau)
$$

The transformation formulas for the theta functions (see [7, Chapter V, Theorem 9, Corollary 1]) provide a connection with the Gaussian function $G_{\lambda}(z)$. In particular, we have

$$
\begin{aligned}
\sum_{n=-\infty}^{\infty}(-1)^{n} G_{\lambda}(n-v) & =\lambda^{-\frac{1}{2}} \theta_{1}\left(v, i \lambda^{-1}\right), \\
\sum_{n=-\infty}^{\infty} G_{\lambda}\left(n+\frac{1}{2}-v\right) & =\lambda^{-\frac{1}{2}} \theta_{2}\left(v, i \lambda^{-1}\right), \\
\sum_{n=-\infty}^{\infty} G_{\lambda}(n-v) & =\lambda^{-\frac{1}{2}} \theta_{3}\left(v, i \lambda^{-1}\right) .
\end{aligned}
$$

Our first main result identifies the entire function $K_{\lambda}(z)$ as the unique best approximation to $G_{\lambda}(z)$ on $\mathbb{R}$ among all entire functions of exponential type at most $\pi$.

Theorem 1. Let $F(z)$ be an entire function of exponential type at most $\pi$. Then

$$
\lambda^{-\frac{1}{2}} \int_{-\frac{1}{2}}^{\frac{1}{2}} \theta_{1}\left(u, i \lambda^{-1}\right) d u \leq \int_{-\infty}^{\infty}\left|G_{\lambda}(x)-F(x)\right| d x,
$$

and there is equality in (1.13) if and only if $F(z)=K_{\lambda}(z)$.

Next we consider the problem of minorizing $G_{\lambda}(z)$ on $\mathbb{R}$ by a real entire function of exponential type at most $2 \pi$.

Theorem 2. Let $F(z)$ be a real entire function of exponential type at most $2 \pi$ such that

$$
F(x) \leq G_{\lambda}(x)
$$


for all real $x$. Then

$$
\int_{-\infty}^{\infty} F(x) d x \leq \lambda^{-\frac{1}{2}} \theta_{2}\left(0, i \lambda^{-1}\right)
$$

and there is equality in (1.14) if and only if $F(z)=L_{\lambda}(z)$.

Here is the analogous result for the problem of majorizing $G_{\lambda}(z)$ on $\mathbb{R}$ by a real entire function of exponential type at most $2 \pi$.

Theorem 3. Let $F(z)$ be a real entire function of exponential type at most $2 \pi$ such that

$$
G_{\lambda}(x) \leq F(x)
$$

for all real $x$. Then

$$
\lambda^{-\frac{1}{2}} \theta_{3}\left(0, i \lambda^{-1}\right) \leq \int_{-\infty}^{\infty} F(x) d x
$$

and there is equality in (1.15) if and only if $F(z)=M_{\lambda}(z)$.

It follows from Theorem 1 that for $\delta>0$, the entire function $z \mapsto K_{\lambda \delta^{-2}}(\delta z)$ is the unique best $L^{1}$-approximation to $G_{\lambda}(x)$ by an entire function of exponential type $\pi \delta$. In a similar manner, using Theorem 2 and Theorem 3 , one can check that the real entire functions $z \mapsto L_{\lambda \delta^{-2}}(\delta z)$ and $z \mapsto M_{\lambda \delta^{-2}}(\delta z)$ are the unique extremal minorant and majorant, respectively, of exponential type $2 \pi \delta$ for the function $G_{\lambda}(x)$.

The entire function $K_{\lambda}(z)$ has exponential type $\pi$, and the restriction $x \mapsto K_{\lambda}(x)$ of this function to $\mathbb{R}$ is clearly integrable. It follows that the Fourier transform

$$
\widehat{K}_{\lambda}(t)=\int_{-\infty}^{\infty} K_{\lambda}(x) e(-x t) \mathrm{d} x
$$

is a continuous function on $\mathbb{R}$, and is supported on the compact interval $\left[-\frac{1}{2}, \frac{1}{2}\right]$. The entire functions $L_{\lambda}(z)$ and $M_{\lambda}(z)$ have exponential type $2 \pi$, and the restrictions of these functions to $\mathbb{R}$ are both integrable. Hence their Fourier transforms

$$
\widehat{L}_{\lambda}(t)=\int_{-\infty}^{\infty} L_{\lambda}(x) e(-x t) \mathrm{d} x \quad \text { and } \quad \widehat{M}_{\lambda}(t)=\int_{-\infty}^{\infty} M_{\lambda}(x) e(-x t) \mathrm{d} x
$$

are both continuous, and both Fourier transforms are supported on the compact interval $[-1,1]$. These Fourier transforms can be given explicitly in terms of the theta functions.

Theorem 4. If $-\frac{1}{2} \leq t \leq \frac{1}{2}$, then the Fourier transform $t \mapsto \widehat{K}_{\lambda}(t)$ is given by

$$
\widehat{K}_{\lambda}(t)=\theta_{1}(t, i \lambda) \text {. }
$$

If $-1 \leq t \leq 1$, then the Fourier transforms $t \mapsto \widehat{L}_{\lambda}(t)$ and $t \mapsto \widehat{M}_{\lambda}(t)$ are given by

$$
\widehat{L}_{\lambda}(t)=(1-|t|) \theta_{1}(t, i \lambda)-(2 \pi)^{-1} \lambda \operatorname{sgn}(t) \frac{\partial \theta_{1}}{\partial t}(t, i \lambda)
$$

and

$$
\widehat{M}_{\lambda}(t)=(1-|t|) \theta_{3}(t, i \lambda)-(2 \pi)^{-1} \lambda \operatorname{sgn}(t) \frac{\partial \theta_{3}}{\partial t}(t, i \lambda) .
$$


Theorem 4 plainly provides an alternative representation for each of the entire functions $K_{\lambda}(z), L_{\lambda}(z)$, and $M_{\lambda}(z)$. Because $t \mapsto \widehat{K}_{\lambda}(t)$ is continuous and has compact support, the Fourier inversion formula and (1.16) imply that

$$
K_{\lambda}(z)=\int_{-\infty}^{\infty} \widehat{K}_{\lambda}(t) e(z t) \mathrm{d} t=\int_{-\frac{1}{2}}^{\frac{1}{2}} \theta_{1}(t, i \lambda) e(z t) \mathrm{d} t
$$

for all complex $z$. Analogous representations hold for the functions $L_{\lambda}(z)$ and $M_{\lambda}(z)$ using (1.17) and (1.18).

The extremal entire functions that we have identified here can be used to determine corresponding extremal trigonometric polynomials associated to the theta functions. Let $N$ be a non-negative integer. We define

$$
\begin{aligned}
k_{\lambda, N}(x) & =\lambda^{\frac{1}{2}} \sum_{n=-\infty}^{\infty} K_{(2 N+2)^{-2} \lambda}((2 N+2)(x+n)) \\
& =\lambda^{\frac{1}{2}}(2 N+2)^{-1} \sum_{n=-N}^{N} \widehat{K}_{(2 N+2)^{-2} \lambda}\left(\frac{n}{2 N+2}\right) e(n x),
\end{aligned}
$$

where the equality of these sums follows from the Poisson summation formula and the fact that $\widehat{K}_{(2 N+2)^{-2} \lambda}(t)=0$ for $\frac{1}{2} \leq|t|$. In particular, $k_{\lambda, N}(x)$ is a trigonometric polynomial of degree $N$ defined on the quotient group $\mathbb{R} / \mathbb{Z}$. We will show that this trigonometric polynomial is the best approximation to the theta function $x \mapsto$ $\theta_{3}\left(x, i \lambda^{-1}\right)$ in $L^{1}$-norm on $\mathbb{R} / \mathbb{Z}$.

Theorem 5. Let $p(x)$ be a trigonometric polynomial of degree at most $N$ defined on $\mathbb{R} / \mathbb{Z}$. Then

$$
\int_{-\frac{1}{2}}^{\frac{1}{2}} \theta_{1}\left(u, i \lambda^{-1}(2 N+2)^{2}\right) d u \leq \int_{\mathbb{R} / \mathbb{Z}}\left|\theta_{3}\left(x, i \lambda^{-1}\right)-p(x)\right| d x,
$$

and there is equality in (1.20) if and only if $p(x)=k_{\lambda, N}(x)$.

In a similar manner, we define

$$
\begin{aligned}
l_{\lambda, N}(x) & =\lambda^{\frac{1}{2}} \sum_{n=-\infty}^{\infty} L_{(N+1)^{-2} \lambda}((N+1)(x+n)) \\
& =\lambda^{\frac{1}{2}}(N+1)^{-1} \sum_{n=-N}^{N} \widehat{L}_{(N+1)^{-2} \lambda}\left(\frac{n}{N+1}\right) e(n x)
\end{aligned}
$$

and

$$
\begin{aligned}
m_{\lambda, N}(x) & =\lambda^{\frac{1}{2}} \sum_{n=-\infty}^{\infty} M_{(N+1)^{-2} \lambda}((N+1)(x+n)) \\
& =\lambda^{\frac{1}{2}}(N+1)^{-1} \sum_{n=-N}^{N} \widehat{M}_{(N+1)^{-2} \lambda}\left(\frac{n}{N+1}\right) e(n x) .
\end{aligned}
$$

Again the identities in (1.21) and (1.22) follow from the Poisson summation formula and the fact that $\widehat{L}_{(N+1)^{-2} \lambda}(t)=\widehat{M}_{(N+1)^{-2} \lambda}(t)=0$ for $1 \leq|t|$. Both of the functions $l_{\lambda, N}(x)$ and $m_{\lambda, N}(x)$ are real valued trigonometric polynomials of degree 
$N$ defined on the quotient group $\mathbb{R} / \mathbb{Z}$. It follows from (1.4) and (1.12) that they satisfy the inequality

$$
l_{\lambda, N}(x) \leq \theta_{3}\left(x, i \lambda^{-1}\right) \leq m_{\lambda, N}(x)
$$

at each point $x$ in $\mathbb{R} / \mathbb{Z}$. We will prove that these trigonometric polynomials are the extreme minorant and majorant for the function $x \mapsto \theta_{3}\left(x, i \lambda^{-1}\right)$ on $\mathbb{R} / \mathbb{Z}$.

Theorem 6. If $q(x)$ is a real valued trigonometric polynomial of degree at most $N$ such that

$$
q(x) \leq \theta_{3}\left(x, i \lambda^{-1}\right)
$$

at each point $x$ in $\mathbb{R} / \mathbb{Z}$, then

$$
\int_{\mathbb{R} / \mathbb{Z}} q(x) d x \leq \theta_{2}\left(0, i \lambda^{-1}(N+1)^{2}\right) .
$$

Moreover, there is equality in (1.25) if and only if $q(x)=l_{\lambda, N}(x)$. If $r(x)$ is a real valued trigonometric polynomial of degree at most $N$ such that

$$
\theta_{3}\left(x, i \lambda^{-1}\right) \leq r(x)
$$

at each point $x$ in $\mathbb{R} / \mathbb{Z}$, then

$$
\theta_{3}\left(0, i \lambda^{-1}(N+1)^{2}\right) \leq \int_{\mathbb{R} / \mathbb{Z}} r(x) d x .
$$

Moreover, there is equality in (1.27) if and only if $r(x)=m_{\lambda, N}(x)$.

\section{INTEGRAL REPRESENTATIONS}

In this section we establish several representations for combinations of Gaussian functions that will be used in the proofs of the previous theorems.

Lemma 7. Let $z$ and $w$ be distinct complex numbers. Then we have

$$
\begin{aligned}
\frac{G_{\lambda}(z)-G_{\lambda}(w)}{z-w}=2 \pi \lambda^{\frac{3}{2}} \int_{-\infty}^{0} \int_{-\infty}^{0} e^{-2 \pi \lambda t u} G_{\lambda}(z-t) G_{\lambda}(w-u) d u d t \\
-2 \pi \lambda^{\frac{3}{2}} \int_{0}^{\infty} \int_{0}^{\infty} e^{-2 \pi \lambda t u} G_{\lambda}(z-t) G_{\lambda}(w-u) d u d t
\end{aligned}
$$

Proof. It suffices to prove the identity (2.1) for $\lambda=1$; then the general case will follow from an elementary change of variables. Therefore we simplify our notation and write $G(z)=G_{1}(z)$. We note that $G(z)$ satisfies the identity

$$
G(z)^{-1}=\int_{-\infty}^{\infty} e^{2 \pi z t} G(t) \mathrm{d} t
$$

for all complex numbers $z$, and the identity

$$
G(z) G(w) e^{2 \pi z w}=G(z-w)
$$

for all pairs of complex numbers $z$ and $w$. From (2.2) we get

$$
\begin{aligned}
\frac{G(z)-G(w)}{z-w} & =G(z) G(w)\left\{\frac{G(w)^{-1}-G(z)^{-1}}{z-w}\right\} \\
& =G(z) G(w)(z-w)^{-1} \int_{-\infty}^{\infty}\left\{e^{2 \pi w t}-e^{2 \pi z t}\right\} G(t) \mathrm{d} t .
\end{aligned}
$$


Then using Fubini's theorem we find that

$$
\begin{aligned}
(z-w)^{-1} \int_{-\infty}^{\infty}\left\{e^{2 \pi w t}-e^{2 \pi z t}\right\} G(t) \mathrm{d} t & \\
= & 2 \pi \int_{-\infty}^{0}\left\{\int_{t}^{0} e^{2 \pi(z-w) u} \mathrm{~d} u\right\} e^{2 \pi w t} G(t) \mathrm{d} t \\
& \quad-2 \pi \int_{0}^{\infty}\left\{\int_{0}^{t} e^{2 \pi(z-w) u} \mathrm{~d} u\right\} e^{2 \pi w t} G(t) \mathrm{d} t \\
= & 2 \pi \int_{-\infty}^{0}\left\{\int_{-\infty}^{u} e^{2 \pi w t} G(t) \mathrm{d} t\right\} e^{2 \pi(z-w) u} \mathrm{~d} u \\
& \quad-2 \pi \int_{0}^{\infty}\left\{\int_{u}^{\infty} e^{2 \pi w t} G(t) \mathrm{d} t\right\} e^{2 \pi(z-w) u} \mathrm{~d} u \\
= & 2 \pi \int_{-\infty}^{0}\left\{\int_{-\infty}^{0} e^{2 \pi w(t+u)} G(t+u) \mathrm{d} t\right\} e^{2 \pi(z-w) u} \mathrm{~d} u \\
& -2 \pi \int_{0}^{\infty}\left\{\int_{0}^{\infty} e^{2 \pi w(t+u)} G(t+u) \mathrm{d} t\right\} e^{2 \pi(z-w) u} \mathrm{~d} u \\
= & 2 \pi \int_{-\infty}^{0} \int_{-\infty}^{0} e^{2 \pi(w t+z u)} G(t+u) \mathrm{d} t \mathrm{~d} u \\
& \quad-2 \pi \int_{0}^{\infty} \int_{0}^{\infty} e^{2 \pi(w t+z u)} G(t+u) \mathrm{d} t \mathrm{~d} u
\end{aligned}
$$

Next we apply (2.3) twice and get

$$
\begin{aligned}
G(z) G(w) e^{2 \pi(w t+z u)} G(t+u) & =G(z) G(w) G(u) G(t) e^{-2 \pi t u+2 \pi w t+2 \pi z u} \\
& =G(z-u) G(w-t) e^{-2 \pi t u} .
\end{aligned}
$$

Then we combine (2.4), (2.5) and (2.6) to obtain the special case

$$
\begin{aligned}
\frac{G(z)-G(w)}{z-w}=2 \pi & \int_{-\infty}^{0} \int_{-\infty}^{0} e^{-2 \pi t u} G(z-t) G(w-u) \mathrm{d} u \mathrm{~d} t \\
& -2 \pi \int_{0}^{\infty} \int_{0}^{\infty} e^{-2 \pi t u} G(z-t) G(w-u) \mathrm{d} u \mathrm{~d} t
\end{aligned}
$$

The more general identity (2.1) follows by replacing $z$ with $\lambda^{\frac{1}{2}} z$, by replacing $w$ with $\lambda^{\frac{1}{2}} w$, and by making a corresponding change of variables in each integral on the right of (2.7).

Lemma 8. Let $z$ and $w$ be distinct complex numbers. Then we have

$$
\begin{aligned}
& \frac{G_{\lambda}(z)}{(z-w)^{2}}-\frac{G_{\lambda}(w)}{(z-w)^{2}}-\frac{G_{\lambda}^{\prime}(w)}{z-w} \\
& =(2 \pi)^{2} \lambda^{\frac{5}{2}} \int_{-\infty}^{0} \int_{-\infty}^{0} t e^{-2 \pi \lambda t u} G_{\lambda}(z-t)\left\{G_{\lambda}(w)-G_{\lambda}(w-u)\right\} d u d t \\
& \quad-(2 \pi)^{2} \lambda^{\frac{5}{2}} \int_{0}^{\infty} \int_{0}^{\infty} t e^{-2 \pi \lambda t u} G_{\lambda}(z-t)\left\{G_{\lambda}(w)-G_{\lambda}(w-u)\right\} d u d t .
\end{aligned}
$$


Proof. We differentiate both sides of (2.1) with respect to $w$ and obtain the identity

$$
\begin{aligned}
& \frac{G_{\lambda}(z)}{(z-w)^{2}}-\frac{G_{\lambda}(w)}{(z-w)^{2}}-\frac{G_{\lambda}^{\prime}(w)}{z-w} \\
& =2 \pi \lambda^{\frac{3}{2}} \int_{-\infty}^{0} \int_{-\infty}^{0} e^{-2 \pi \lambda t u} G_{\lambda}(z-t) G_{\lambda}^{\prime}(w-u) \mathrm{d} u \mathrm{~d} t \\
& \quad-2 \pi \lambda^{\frac{3}{2}} \int_{0}^{\infty} \int_{0}^{\infty} e^{-2 \pi \lambda t u} G_{\lambda}(z-t) G_{\lambda}^{\prime}(w-u) \mathrm{d} u \mathrm{~d} t .
\end{aligned}
$$

Using integration by parts we get

$$
\begin{aligned}
& \int_{-\infty}^{0} e^{-2 \pi \lambda t u} G_{\lambda}^{\prime}(w-u) \mathrm{d} u \\
&=2 \pi \lambda \int_{-\infty}^{0} t e^{-2 \pi \lambda t u}\left\{G_{\lambda}(w)-G_{\lambda}(w-u)\right\} \mathrm{d} u
\end{aligned}
$$

and

$$
\begin{aligned}
& \int_{0}^{\infty} e^{-2 \pi \lambda t u} G_{\lambda}^{\prime}(w-u) \mathrm{d} u \\
&=2 \pi \lambda \int_{0}^{\infty} t e^{-2 \pi \lambda t u}\left\{G_{\lambda}(w)-G_{\lambda}(w-u)\right\} \mathrm{d} u .
\end{aligned}
$$

The corollary now follows by combining (2.9), (2.10) and (2.11).

In order to apply the identities (1.10), (1.11) and (1.12), we require simple estimates for certain partial sums.

Lemma 9. For all real $u$ and positive integers $N$, we have

$$
\begin{aligned}
\sum_{n=-N-1}^{N}(-1)^{n} G_{\lambda}\left(n+\frac{1}{2}-u\right) & \ll_{\lambda} \min \{1,|u|\}, \\
\sum_{n=-N-1}^{N}\left\{G_{\lambda}\left(n+\frac{1}{2}\right)-G_{\lambda}\left(n+\frac{1}{2}-u\right)\right\} & \ll_{\lambda} \min \{1,|u|\}, \\
\sum_{n=-N}^{N}\left\{G_{\lambda}(n)-G_{\lambda}(n-u)\right\} & \ll_{\lambda} \min \{1,|u|\},
\end{aligned}
$$

where the constant implied by $\ll_{\lambda}$ depends on $\lambda$, but not on $u$ or $N$.

Proof. For each positive integer $N$,

$$
u \mapsto S_{\lambda, N}(u)=\sum_{n=-N-1}^{N}(-1)^{n} G_{\lambda}\left(n+\frac{1}{2}-u\right)
$$

is an odd function of $u$. Hence its derivative is an even function of $u$. Therefore we get

$$
\begin{aligned}
\left|S_{\lambda, N}(u)\right| & =\left|\int_{0}^{u} S_{\lambda, N}^{\prime}(v) \mathrm{d} v\right| \\
& \leq \int_{0}^{|u|}\left\{\sum_{n=-\infty}^{\infty}\left|G_{\lambda}^{\prime}\left(n+\frac{1}{2}-v\right)\right|\right\} \mathrm{d} v \\
& \leq C_{\lambda}|u|,
\end{aligned}
$$


where

$$
C_{\lambda}=\sup _{v \in \mathbb{R}}\left\{\sum_{n=-\infty}^{\infty}\left|G_{\lambda}^{\prime}\left(n+\frac{1}{2}-v\right)\right|\right\}
$$

is obviously finite. We also have

$$
\left|S_{\lambda, N}(u)\right| \leq \sup _{v \in \mathbb{R}}\left\{\sum_{n=-\infty}^{\infty}\left|G_{\lambda}\left(n+\frac{1}{2}-v\right)\right|\right\}<\infty,
$$

and the bound (2.12) follows.

The proofs of (2.13) and (2.14) are very similar.

Lemma 10. For all real $u$ and positive integers $N$, let $S_{\lambda, N}(u)$ be defined by the sum on the left of (2.12). Then for $0<t$ we have

$$
\left|\int_{0}^{\infty} e^{-2 \pi \lambda t u} S_{\lambda, N}(u) d u\right| \leq \lambda^{-\frac{1}{2}}
$$

and for $t<0$ we have

$$
\left|\int_{-\infty}^{0} e^{-2 \pi \lambda t u} S_{\lambda, N}(u) d u\right| \leq \lambda^{-\frac{1}{2}}
$$

Proof. Let $0<t$ and $0<u$. For positive integers $N$ we define

$$
R_{\lambda, N}(u)=\int_{0}^{u} S_{\lambda, N}(v) \mathrm{d} v
$$

Then it follows, using integration by parts, that

$$
\int_{0}^{\infty} e^{-2 \pi \lambda t u} S_{\lambda, N}(u) \mathrm{d} u=2 \pi \lambda t \int_{0}^{\infty} e^{-2 \pi \lambda t u} R_{\lambda, N}(u) \mathrm{d} u .
$$

For $\alpha<\beta$, let

$$
\chi_{\alpha, \beta}(x)=\frac{1}{2} \operatorname{sgn}(\beta-x)+\frac{1}{2} \operatorname{sgn}(x-\alpha)
$$

denote the normalized characteristic function of the the real interval with endpoints $\alpha$ and $\beta$. Using the inequality

$$
\left|\sum_{n=-N-1}^{N}(-1)^{n} \chi_{n+\frac{1}{2}-u, n+\frac{1}{2}}(x)\right| \leq 1,
$$

we find that

$$
\begin{aligned}
\left|R_{\lambda, N}(u)\right| & =\left|\sum_{n=-N-1}^{N}(-1)^{n} \int_{0}^{u} G_{\lambda}\left(n+\frac{1}{2}-v\right) \mathrm{d} v\right| \\
& =\left|\int_{-\infty}^{\infty}\left\{\sum_{n=-N-1}^{N}(-1)^{n} \chi_{n+\frac{1}{2}-u, n+\frac{1}{2}}(w)\right\} G_{\lambda}(w) \mathrm{d} w\right| \\
& \leq \int_{-\infty}^{\infty} G_{\lambda}(w) \mathrm{d} w \\
& =\lambda^{-\frac{1}{2}}
\end{aligned}
$$


Then using (2.17) we get

$$
\begin{aligned}
\left|\int_{0}^{\infty} e^{-2 \pi \lambda t u} S_{\lambda, N}(u) \mathrm{d} u\right| & \leq 2 \pi \lambda t \int_{0}^{\infty} e^{-2 \pi \lambda t u}\left|R_{\lambda, N}(u)\right| \mathrm{d} u \\
& \leq 2 \pi \lambda^{\frac{1}{2}} t \int_{0}^{\infty} e^{-2 \pi \lambda t u} \mathrm{~d} u \\
& =\lambda^{-\frac{1}{2}}
\end{aligned}
$$

This verifies (2.15), and (2.16) follows from (2.15) because $u \mapsto S_{\lambda, N}(u)$ is an odd function.

We have noted that the entire function $z \mapsto G_{\lambda}(z)-K_{\lambda}(z)$ vanishes at each point of the coset $\mathbb{Z}+\frac{1}{2}$. It follows that

$$
z \mapsto \frac{\pi}{\cos \pi z}\left\{G_{\lambda}(z)-K_{\lambda}(z)\right\}
$$

is an entire function.

Lemma 11. For all complex $z$ we have

$$
\begin{aligned}
\frac{\pi}{\cos \pi z} & \left\{G_{\lambda}(z)-K_{\lambda}(z)\right\} \\
& =\pi \lambda \int_{-\infty}^{\infty} \frac{G_{\lambda}(z-t)}{\cosh \pi \lambda t} \int_{-\frac{1}{2}}^{\frac{1}{2}} \cosh 2 \pi \lambda t u \theta_{1}\left(u, i \lambda^{-1}\right) d u d t .
\end{aligned}
$$

Proof. We use the partial fraction expansion

$$
\lim _{N \rightarrow \infty} \sum_{n=-N-1}^{N} \frac{(-1)^{n+1}}{z-n-\frac{1}{2}}=\frac{\pi}{\cos \pi z},
$$

which converges uniformly on compact subsets of $\mathbb{C} \backslash\left\{\mathbb{Z}+\frac{1}{2}\right\}$. Then it follows from (1.1) and (2.19) that

$$
\begin{aligned}
\frac{\pi}{\cos \pi z} & \left\{G_{\lambda}(z)-K_{\lambda}(z)\right\} \\
& =\lim _{N \rightarrow \infty} \sum_{n=-N-1}^{N}(-1)^{n+1}\left\{\frac{G_{\lambda}(z)-G_{\lambda}\left(n+\frac{1}{2}\right)}{z-n-\frac{1}{2}}\right\} .
\end{aligned}
$$

As the function on the left of (2.20) is entire and a compact subset of $\mathbb{C}$ intersects $\mathbb{Z}+\frac{1}{2}$ in finitely many points, we find that the limit on the right of (2.20) converges uniformly on compact subsets of $\mathbb{C}$.

For positive integers $N$ and all real $u$ let

$$
S_{\lambda, N}(u)=\sum_{n=-N-1}^{N}(-1)^{n} G_{\lambda}\left(n+\frac{1}{2}-u\right) .
$$

Then (1.10) implies that

$$
\lim _{N \rightarrow \infty} S_{\lambda, N}(u)=\lambda^{-\frac{1}{2}} \theta_{1}\left(u-\frac{1}{2}, i \lambda^{-1}\right) .
$$


We use the identity (2.1) with $w=n+\frac{1}{2}$ and sum over integers $n$ satisfying $-N-1 \leq n \leq N$. We find that

$$
\begin{aligned}
\sum_{n=-N-1}^{N}(-1)^{n+1}\left\{\frac{G_{\lambda}(z)-G_{\lambda}\left(n+\frac{1}{2}\right)}{z-n-\frac{1}{2}}\right\} & \\
= & 2 \pi \lambda^{\frac{3}{2}} \int_{0}^{\infty} G_{\lambda}(z-t)\left\{\int_{0}^{\infty} e^{-2 \pi \lambda t u} S_{\lambda, N}(u) \mathrm{d} u\right\} \mathrm{d} t \\
& -2 \pi \lambda^{\frac{3}{2}} \int_{-\infty}^{0} G_{\lambda}(z-t)\left\{\int_{-\infty}^{0} e^{-2 \pi \lambda t u} S_{\lambda, N}(u) \mathrm{d} u\right\} \mathrm{d} t .
\end{aligned}
$$

Next we let $N \rightarrow \infty$ on both sides of (2.22). The limit on the left hand side is determined by (2.20). On the right hand side we use (2.15), (2.16), and the dominated convergence theorem to move the limits inside the outer integrals. Then we use (2.12) and the dominated convergence theorem to move the limits inside the inner integrals. Applying (2.21) we arrive at the identity

$$
\begin{aligned}
& \frac{\pi}{\cos \pi z}\left\{G_{\lambda}(z)-K_{\lambda}(z)\right\} \\
&=2 \pi \lambda \int_{0}^{\infty} \int_{0}^{\infty} e^{-2 \pi \lambda t u} G_{\lambda}(z-t) \theta_{1}\left(u-\frac{1}{2}, i \lambda^{-1}\right) \mathrm{d} u \mathrm{~d} t \\
& \quad-2 \pi \lambda \int_{-\infty}^{0} \int_{-\infty}^{0} e^{-2 \pi \lambda t u} G_{\lambda}(z-t) \theta_{1}\left(u-\frac{1}{2}, i \lambda^{-1}\right) \mathrm{d} u \mathrm{~d} t .
\end{aligned}
$$

If $0<t$, then, using (1.8) and the fact that $u \mapsto \theta_{1}\left(u, i \lambda^{-1}\right)$ is an even function, we get

$$
\begin{aligned}
\int_{0}^{\infty} e^{-2 \pi \lambda t u} \theta_{1} & \left(u-\frac{1}{2}, i \lambda^{-1}\right) \mathrm{d} u \\
& =\sum_{m=0}^{\infty} \int_{0}^{1} e^{-2 \pi \lambda t(u+m)} \theta_{1}\left(u+m-\frac{1}{2}, i \lambda^{-1}\right) \mathrm{d} u \\
& =\sum_{m=0}^{\infty}(-1)^{m} e^{-2 \pi \lambda t m} \int_{0}^{1} e^{-2 \pi \lambda t u} \theta_{1}\left(u-\frac{1}{2}, i \lambda^{-1}\right) \mathrm{d} u \\
& =\left\{e^{\pi \lambda t}+e^{-\pi \lambda t}\right\}^{-1} \int_{-\frac{1}{2}}^{\frac{1}{2}} e^{-2 \pi \lambda t u} \theta_{1}\left(u, i \lambda^{-1}\right) \mathrm{d} u \\
& =\{2 \cosh \pi \lambda t\}^{-1} \int_{-\frac{1}{2}}^{\frac{1}{2}} \cosh 2 \pi \lambda t u \theta_{1}\left(u, i \lambda^{-1}\right) \mathrm{d} u .
\end{aligned}
$$

If $t<0$, then in a similar manner we find that

$$
\begin{aligned}
\int_{-\infty}^{0} e^{-2 \pi \lambda t u} \theta_{1}\left(u-\frac{1}{2}, i \lambda^{-1}\right) \mathrm{d} u & \\
= & -\{2 \cosh \pi \lambda t\}^{-1} \int_{-\frac{1}{2}}^{\frac{1}{2}} \cosh 2 \pi \lambda t u \theta_{1}\left(u, i \lambda^{-1}\right) \mathrm{d} u .
\end{aligned}
$$

The identity (2.18) follows now by combining (2.23), (2.24) and (2.25). 
Because $z \mapsto L_{\lambda}(z)$ interpolates both the value of $G_{\lambda}(z)$ and the value of its derivative $G_{\lambda}^{\prime}(z)$ at each point of the coset $\mathbb{Z}+\frac{1}{2}$, the entire function

$$
z \mapsto G_{\lambda}(z)-L_{\lambda}(z)
$$

has a zero of multiplicity at least 2 at each point of $\mathbb{Z}+\frac{1}{2}$. It follows that

$$
z \mapsto\left(\frac{\pi}{\cos \pi z}\right)^{2}\left\{G_{\lambda}(z)-L_{\lambda}(z)\right\}
$$

is an entire function. In a similar manner, we find that

$$
z \mapsto\left(\frac{\pi}{\sin \pi z}\right)^{2}\left\{M_{\lambda}(z)-G_{\lambda}(z)\right\}
$$

is an entire function.

Lemma 12. For all complex $z$ we have

$$
\begin{aligned}
& \left(\frac{\pi}{\cos \pi z}\right)^{2}\left\{G_{\lambda}(z)-L_{\lambda}(z)\right\} \\
& =2 \pi^{2} \lambda^{2} \int_{-\infty}^{\infty} \frac{t G_{\lambda}(z-t)}{\sinh \pi \lambda t} \int_{-\frac{1}{2}}^{\frac{1}{2}} e^{-2 \pi \lambda t u}\left\{\theta_{3}\left(u, i \lambda^{-1}\right)-\theta_{3}\left(\frac{1}{2}, i \lambda^{-1}\right)\right\} d u d t
\end{aligned}
$$

and

$$
\begin{aligned}
& \left(\frac{\pi}{\sin \pi z}\right)^{2}\left\{M_{\lambda}(z)-G_{\lambda}(z)\right\} \\
& =2 \pi^{2} \lambda^{2} \int_{-\infty}^{\infty} \frac{t G_{\lambda}(z-t)}{\sinh \pi \lambda t} \int_{-\frac{1}{2}}^{\frac{1}{2}} e^{-2 \pi \lambda t u}\left\{\theta_{2}\left(\frac{1}{2}, i \lambda^{-1}\right)-\theta_{2}\left(u, i \lambda^{-1}\right)\right\} d u d t
\end{aligned}
$$

Proof. In order to establish (2.26) we use the partial fraction expansion

$$
\lim _{N \rightarrow \infty} \sum_{n=-N-1}^{N} \frac{1}{\left(z-n-\frac{1}{2}\right)^{2}}=\left(\frac{\pi}{\cos \pi z}\right)^{2},
$$

which converges uniformly on compact subsets of $\mathbb{C} \backslash\left\{\mathbb{Z}+\frac{1}{2}\right\}$. Then it follows from (1.2) and (2.28) that

$$
\begin{aligned}
\left(\frac{\pi}{\cos \pi z}\right)^{2}\left\{G_{\lambda}(z)-L_{\lambda}(z)\right\} & \\
= & \lim _{N \rightarrow \infty} \sum_{n=-N-1}^{N}\left\{\frac{G_{\lambda}(z)}{\left(z-n-\frac{1}{2}\right)^{2}}-\frac{G_{\lambda}\left(n+\frac{1}{2}\right)}{\left(z-n-\frac{1}{2}\right)^{2}}-\frac{G_{\lambda}^{\prime}\left(n+\frac{1}{2}\right)}{z-n-\frac{1}{2}}\right\} .
\end{aligned}
$$

As in the proof of Lemma 11] the limit on the right of (2.29) converges uniformly on compact subsets of $\mathbb{C}$.

For positive integers $N$ and all real $u$ let

$$
T_{\lambda, N}(u)=\sum_{n=-N-1}^{N}\left\{G_{\lambda}\left(n+\frac{1}{2}\right)-G_{\lambda}\left(n+\frac{1}{2}-u\right)\right\} .
$$

From (1.11) we conclude that

$$
\lim _{N \rightarrow \infty} T_{\lambda, N}(u)=\lambda^{-\frac{1}{2}}\left\{\theta_{2}\left(0, i \lambda^{-1}\right)-\theta_{2}\left(u, i \lambda^{-1}\right)\right\} .
$$


We apply the identity (2.8) with $w=n+\frac{1}{2}$ and sum over integers $n$ satisfying $-N-1 \leq n \leq N$. We get

$$
\begin{aligned}
\sum_{n=-N-1}^{N}\left\{\frac{G_{\lambda}(z)}{\left(z-n-\frac{1}{2}\right)^{2}}-\frac{G_{\lambda}\left(n+\frac{1}{2}\right)}{\left(z-n-\frac{1}{2}\right)^{2}}-\frac{G_{\lambda}^{\prime}\left(n+\frac{1}{2}\right)}{z-n-\frac{1}{2}}\right\} \\
=(2 \pi)^{2} \lambda^{\frac{5}{2}} \int_{-\infty}^{0} \int_{-\infty}^{0} t e^{-2 \pi \lambda t u} G_{\lambda}(z-t) T_{\lambda, N}(u) \mathrm{d} u \mathrm{~d} t \\
\quad-(2 \pi)^{2} \lambda^{\frac{5}{2}} \int_{0}^{\infty} \int_{0}^{\infty} t e^{-2 \pi \lambda t u} G_{\lambda}(z-t) T_{\lambda, N}(u) \mathrm{d} u \mathrm{~d} t
\end{aligned}
$$

As in the proof of Lemma 11, we let $N \rightarrow \infty$ on both sides of (2.31). The limit on the left hand side is determined by (2.29). On the right hand side we use (2.13), the dominated convergence theorem and (2.30). In this way we obtain the identity

$$
\begin{aligned}
& \left(\frac{\pi}{\cos \pi z}\right)^{2}\left\{G_{\lambda}(z)-L_{\lambda}(z)\right\} \\
& =(2 \pi \lambda)^{2} \int_{-\infty}^{0} \int_{-\infty}^{0} t e^{-2 \pi \lambda t u} G_{\lambda}(z-t)\left\{\theta_{2}\left(0, i \lambda^{-1}\right)-\theta_{2}\left(u, i \lambda^{-1}\right)\right\} \mathrm{d} u \mathrm{~d} t \\
& \quad-(2 \pi \lambda)^{2} \int_{0}^{\infty} \int_{0}^{\infty} t e^{-2 \pi \lambda t u} G_{\lambda}(z-t)\left\{\theta_{2}\left(0, i \lambda^{-1}\right)-\theta_{2}\left(u, i \lambda^{-1}\right)\right\} \mathrm{d} u \mathrm{~d} t .
\end{aligned}
$$

If $0<t$, then, using that $v \mapsto \theta_{2}(v, \tau)$ has period 1 and (1.9), we get

$$
\begin{aligned}
\int_{0}^{\infty} & e^{-2 \pi \lambda t u}\left\{\theta_{2}\left(0, i \lambda^{-1}\right)-\theta_{2}\left(u, i \lambda^{-1}\right)\right\} \mathrm{d} u \\
& =\sum_{m=0}^{\infty} \int_{0}^{1} e^{-2 \pi \lambda t(u+m)}\left\{\theta_{2}\left(0, i \lambda^{-1}\right)-\theta_{2}\left(u+m, i \lambda^{-1}\right)\right\} \mathrm{d} u \\
& =\left\{1-e^{-2 \pi \lambda t}\right\}^{-1} \int_{0}^{1} e^{-2 \pi \lambda t u}\left\{\theta_{2}\left(0, i \lambda^{-1}\right)-\theta_{2}\left(u, i \lambda^{-1}\right)\right\} \mathrm{d} u \\
& =\{2 \sinh \pi \lambda t\}^{-1} \int_{-\frac{1}{2}}^{\frac{1}{2}} e^{-2 \pi \lambda t u}\left\{\theta_{3}\left(\frac{1}{2}, i \lambda^{-1}\right)-\theta_{3}\left(u, i \lambda^{-1}\right)\right\} \mathrm{d} u .
\end{aligned}
$$

If $t<0$, then in a similar manner we find that

$$
\begin{aligned}
& \int_{-\infty}^{0} e^{-2 \pi \lambda t u}\left\{\theta_{2}\left(0, i \lambda^{-1}\right)-\theta_{2}\left(u, i \lambda^{-1}\right)\right\} \mathrm{d} u \\
& \quad=-\{2 \sinh \pi \lambda t\}^{-1} \int_{-\frac{1}{2}}^{\frac{1}{2}} e^{-2 \pi \lambda t u}\left\{\theta_{3}\left(\frac{1}{2}, i \lambda^{-1}\right)-\theta_{3}\left(u, i \lambda^{-1}\right)\right\} \mathrm{d} u
\end{aligned}
$$

The identity (2.26) now follows by combining (2.32), (2.33) and (2.34).

The proof of (2.27) proceeds along the same lines using (1.12) and (2.14). We leave the details to the reader.

Corollary 13. For all real values of $x$ we have

$$
0<\left(\frac{\pi}{\cos \pi x}\right)^{2}\left\{G_{\lambda}(x)-L_{\lambda}(x)\right\}
$$


and

$$
0<\left(\frac{\pi}{\sin \pi x}\right)^{2}\left\{M_{\lambda}(x)-G_{\lambda}(x)\right\} .
$$

In particular, the inequality (1.4) holds for all real $x$.

Proof. For real $u$ the periodic function $u \mapsto \theta_{3}\left(u, i \lambda^{-1}\right)$ takes its maximum value at $u=0$ and its minimum values at $u=\frac{1}{2}$. Therefore the function

$$
t \mapsto \int_{-\frac{1}{2}}^{\frac{1}{2}} e^{-2 \pi \lambda t u}\left\{\theta_{3}\left(u, i \lambda^{-1}\right)-\theta_{3}\left(\frac{1}{2}, i \lambda^{-1}\right)\right\} \mathrm{d} u,
$$

which appears in the integrand on the right of (2.26), is positive for all real values of $t$. This plainly verifies the inequality (2.35).

In a similar manner using (1.9), the periodic function $u \mapsto \theta_{2}\left(u, i \lambda^{-1}\right)$ takes its maximum value at $u=\frac{1}{2}$ and its minimum value at $u=0$. Hence the function

$$
t \mapsto \int_{-\frac{1}{2}}^{\frac{1}{2}} e^{-2 \pi \lambda t u}\left\{\theta_{2}\left(\frac{1}{2}, i \lambda^{-1}\right)-\theta_{2}\left(u, i \lambda^{-1}\right)\right\} \mathrm{d} u,
$$

which appears in the integrand on the right of (2.27), is positive for all real values of $t$. This establishes the inequality (2.36).

\section{Proof of Theorem 1}

We recall that $v \mapsto \theta_{1}(v, i \lambda)$ is an entire function of the complex variable $v$. The product formula for this theta function (see [7, Chapter V, Theorem 6]) provides the representation

$$
\begin{aligned}
\theta_{1}\left(v, i \lambda^{-1}\right)=2 e^{-\pi(4 \lambda)^{-1}} & \prod_{l=1}^{\infty}\left(1-e^{-2 \pi \lambda^{-1} l}\right) \cos \pi v \\
& \prod_{m=1}^{\infty}\left(1+e^{-2 \pi \lambda^{-1} m+2 \pi i v}\right) \prod_{n=1}^{\infty}\left(1+e^{-2 \pi \lambda^{-1} n-2 \pi i v}\right) .
\end{aligned}
$$

It follows from (3.1) that the only real zeros of $\theta_{1}\left(v, i \lambda^{-1}\right)$ are zeros of $\cos \pi v$. That is, the only real zeros are simple zeros at the points of $\mathbb{Z}+\frac{1}{2}$. Because $\theta_{1}\left(0, i \lambda^{-1}\right)>0$, it follows that $\theta_{1}\left(u, i \lambda^{-1}\right)>0$ for all real values of $u$ in the open interval $-\frac{1}{2}<u<\frac{1}{2}$. This implies that the integral on the left of (1.13) is positive. Also, the integral on the right of (2.18) is positive for real values of $z=x$. Alternatively, we have

$$
\frac{\pi}{\cos \pi x}\left\{G_{\lambda}(x)-K_{\lambda}(x)\right\}>0
$$

for all real $x$, and therefore

$$
\operatorname{sgn}\left\{G_{\lambda}(x)-K_{\lambda}(x)\right\}=\operatorname{sgn}(\cos \pi x)
$$

for all real $x$.

From the series expansion (1.5) we find that

$$
\lambda^{-\frac{1}{2}} \int_{-\frac{1}{2}}^{\frac{1}{2}} \theta_{1}\left(u, i \lambda^{-1}\right) \mathrm{d} u=\frac{1}{\pi} \sum_{n=-\infty}^{\infty} \frac{(-1)^{n}}{n+\frac{1}{2}} \widehat{G}_{\lambda}\left(n+\frac{1}{2}\right),
$$


where

$$
\widehat{G}_{\lambda}(t)=\lambda^{-\frac{1}{2}} e^{-\pi \lambda^{-1} t^{2}}=\int_{-\infty}^{\infty} G_{\lambda}(x) e(-x t) \mathrm{d} x
$$

is the Fourier transform of $G_{\lambda}(x)$ on $\mathbb{R}$. Now let $F(z)$ be an entire function of exponential type at most $\pi$. Without loss of generality we may assume that

$$
\int_{-\infty}^{\infty}\left|G_{\lambda}(x)-F(x)\right| \mathrm{d} x<\infty .
$$

It follows that $F$ is integrable on $\mathbb{R}$, and therefore the Fourier transform

$$
t \mapsto \widehat{F}(t)=\int_{-\infty}^{\infty} F(x) e(-t x) \mathrm{d} x
$$

is continuous on $\mathbb{R}$ and supported on $\left[-\frac{1}{2}, \frac{1}{2}\right]$. The function

$$
x \mapsto \operatorname{sgn}(\cos \pi x)
$$

is periodic on $\mathbb{R}$ with period 2 and has the Fourier expansion

$$
\operatorname{sgn}(\cos \pi x)=\lim _{N \rightarrow \infty} \frac{1}{\pi} \sum_{n=-N-1}^{N} \frac{(-1)^{n}}{n+\frac{1}{2}} e\left(\left(n+\frac{1}{2}\right) x\right) .
$$

Moreover, the partial sums on the right of (3.4) are uniformly bounded, and therefore

$$
\begin{aligned}
\int_{-\infty}^{\infty} \operatorname{sgn}(\cos \pi x)\left\{G_{\lambda}(x)-F(x)\right\} \mathrm{d} x \\
=\lim _{N \rightarrow \infty} \frac{1}{\pi} \sum_{n=-N-1}^{N} \frac{(-1)^{n}}{n+\frac{1}{2}} \int_{-\infty}^{\infty}\left\{G_{\lambda}(x)-F(x)\right\} e\left(\left(n+\frac{1}{2}\right) x\right) \mathrm{d} x \\
=\lim _{N \rightarrow \infty} \frac{1}{\pi} \sum_{n=-N-1}^{N} \frac{(-1)^{n}}{n+\frac{1}{2}}\left\{\widehat{G}_{\lambda}\left(-n-\frac{1}{2}\right)-\widehat{F}\left(-n-\frac{1}{2}\right)\right\} \\
=\frac{1}{\pi} \sum_{n=-\infty}^{\infty} \frac{(-1)^{n}}{n+\frac{1}{2}} \widehat{G}_{\lambda}\left(n+\frac{1}{2}\right) .
\end{aligned}
$$

It is clear from (3.3) and (3.5) that

$$
\lambda^{-\frac{1}{2}} \int_{-\frac{1}{2}}^{\frac{1}{2}} \theta_{1}\left(u, i \lambda^{-1}\right) \mathrm{d} u \leq \int_{-\infty}^{\infty}\left|G_{\lambda}(x)-F(x)\right| \mathrm{d} x,
$$

and this verifies (1.13). Then (3.2), (3.3) and (3.5) lead to the identity

$$
\begin{aligned}
\lambda^{-\frac{1}{2}} \int_{-\frac{1}{2}}^{\frac{1}{2}} \theta_{1}\left(u, i \lambda^{-1}\right) \mathrm{d} u & =\int_{-\infty}^{\infty} \operatorname{sgn}(\cos \pi x)\left\{G_{\lambda}(x)-K_{\lambda}(x)\right\} \mathrm{d} x \\
& =\int_{-\infty}^{\infty}\left|G_{\lambda}(x)-K_{\lambda}(x)\right| \mathrm{d} x .
\end{aligned}
$$

Plainly (3.7) shows that there is equality in the inequality (1.13) in case $F(z)=$ $K_{\lambda}(z)$. 
Finally, we assume that $F(z)$ is an entire function of exponential type at most $\pi$ for which there is equality in the inequality (1.13). Then (3.3) and (3.5) imply that

$$
\int_{-\infty}^{\infty} \operatorname{sgn}(\cos \pi x)\left\{G_{\lambda}(x)-F(x)\right\} \mathrm{d} x=\int_{-\infty}^{\infty}\left|G_{\lambda}(x)-F(x)\right| \mathrm{d} x .
$$

As $x \mapsto G_{\lambda}(x)-F(x)$ is continuous, we conclude from (3.8) that

$$
\operatorname{sgn}(\cos \pi x)\left\{G_{\lambda}(x)-F(x)\right\}=\left|G_{\lambda}(x)-F(x)\right|
$$

for all real $x$. This implies that

$$
G_{\lambda}\left(n+\frac{1}{2}\right)=K_{\lambda}\left(n+\frac{1}{2}\right)=F\left(n+\frac{1}{2}\right)
$$

for each integer $n$. Therefore

$$
z \mapsto K_{\lambda}(z)-F(z)
$$

is an entire function of exponential type at most $\pi$ and takes the value zero at each point of the set $\mathbb{Z}+\frac{1}{2}$. From basic interpolation theorems for entire functions of exponential type (see [37, Vol. II, p. 275]), we conclude that the entire function (3.9) is identically zero. This completes the proof of Theorem 1.

\section{Proofs of Theorems 2 and 3}

Let $F(z)$ be an entire function of exponential type at most $2 \pi$ such that

$$
F(x) \leq G_{\lambda}(x)
$$

for all real $x$. Clearly we may assume that $x \mapsto F(x)$ is integrable on $\mathbb{R}$, for if not, then (1.14) is trivial. Using [14, Lemma 4], (1.11) and (4.1), we find that

$$
\begin{aligned}
\int_{-\infty}^{\infty} F(x) \mathrm{d} x & =\lim _{N \rightarrow \infty} \sum_{n=-N}^{N}\left(1-\frac{|n|}{N+1}\right) F(n+v) \\
& \leq \lim _{N \rightarrow \infty} \sum_{n=-N}^{N}\left(1-\frac{|n|}{N+1}\right) G_{\lambda}(n+v) \\
& =\lambda^{-\frac{1}{2}} \theta_{2}\left(\frac{1}{2}-v, i \lambda^{-1}\right)
\end{aligned}
$$

for all real $v$. We have already noted that $v \mapsto \theta_{2}\left(\frac{1}{2}-v, i \lambda^{-1}\right)$ takes its minimum value at $v=\frac{1}{2}$. Hence (4.2) implies that

$$
\int_{-\infty}^{\infty} F(x) \mathrm{d} x \leq \lambda^{-\frac{1}{2}} \theta_{2}\left(0, i \lambda^{-1}\right),
$$

and this proves (1.14).

In Corollary 13 we proved that $F(z)=L_{\lambda}(z)$ satisfies the inequality (4.1) for all real $x$. In this special case there is equality in the inequality (4.2) when $v=\frac{1}{2}$. Thus we have

$$
\int_{-\infty}^{\infty} L_{\lambda}(x) \mathrm{d} x=\lambda^{-\frac{1}{2}} \theta_{2}\left(0, i \lambda^{-1}\right)
$$

Now assume that $F(z)$ is an entire function of exponential type at most $2 \pi$ that satisfies (4.1) for all real $x$, and assume that there is equality in the inequality (4.2). It follows that $v=\frac{1}{2}$ and

$$
F\left(n+\frac{1}{2}\right)=G_{\lambda}\left(n+\frac{1}{2}\right)
$$


for all integers $n$. Then from (4.1) we also get

$$
F^{\prime}\left(n+\frac{1}{2}\right)=G_{\lambda}^{\prime}\left(n+\frac{1}{2}\right)
$$

for all integers $n$. Of course this shows that the entire function

$$
z \mapsto F(z)-L_{\lambda}(z)
$$

has exponential type at most $2 \pi$, vanishes at each point of $\mathbb{Z}+\frac{1}{2}$, and its derivative also vanishes at each point of $\mathbb{Z}+\frac{1}{2}$. By a second application of [14, Lemma 4] we conclude that the entire function (4.4) is identically zero. This proves Theorem 2, and Theorem 3 can be proved by the same type of argument.

\section{Proof of Theorem 4}

The partial sums for the series (1.5) defining $t \mapsto \theta_{1}(t, i \lambda)$ converge absolutely and uniformly for $t$ in $\mathbb{R}$. Therefore we find that

$$
\begin{aligned}
\int_{-\frac{1}{2}}^{\frac{1}{2}} \theta_{1}(t, i \lambda) e(t z) \mathrm{d} t & =\sum_{n=-\infty}^{\infty} e^{-\pi \lambda\left(n+\frac{1}{2}\right)^{2}} \int_{-\frac{1}{2}}^{\frac{1}{2}} e\left(t\left(z+n+\frac{1}{2}\right)\right) \mathrm{d} t \\
& =\left(\frac{\cos \pi z}{\pi}\right)\left\{\sum_{n=-\infty}^{\infty}(-1)^{n} \frac{e^{-\pi \lambda\left(n+\frac{1}{2}\right)^{2}}}{\left(z+n+\frac{1}{2}\right)}\right\} \\
& =K_{\lambda}(z) .
\end{aligned}
$$

Then (1.16) follows from (5.1) by Fourier inversion. In a similar manner we find that

$$
\begin{aligned}
\int_{-1}^{1}(1-|t|) & \theta_{1}(t, i \lambda) e(t z) \mathrm{d} t \\
& =\sum_{n=-\infty}^{\infty} e^{-\pi \lambda\left(n+\frac{1}{2}\right)^{2}} \int_{-1}^{1}(1-|t|) e\left(t\left(z+n+\frac{1}{2}\right)\right) \mathrm{d} t \\
& =\sum_{-\infty}^{\infty} e^{-\pi \lambda\left(n+\frac{1}{2}\right)^{2}}\left(\frac{\sin \pi\left(z+n+\frac{1}{2}\right)}{\pi\left(z+n+\frac{1}{2}\right)}\right)^{2} \\
& =\left(\frac{\cos \pi z}{\pi}\right)^{2} \sum_{m=-\infty}^{\infty} \frac{G_{\lambda}\left(m+\frac{1}{2}\right)}{\left(z-m-\frac{1}{2}\right)^{2}}
\end{aligned}
$$

and

$$
\begin{aligned}
-(2 \pi)^{-1} \lambda \int_{-1}^{1} & \operatorname{sgn}(t) \frac{\partial \theta_{1}}{\partial t}(t, i \lambda) e(t z) \mathrm{d} t \\
& =-(2 \pi)^{-1} \lambda \sum_{-\infty}^{\infty} e^{-\pi \lambda\left(n+\frac{1}{2}\right)^{2}}\left\{2 \pi i\left(n+\frac{1}{2}\right)\right\} \int_{-1}^{1} \operatorname{sgn}(t) e(t z) \mathrm{d} t \\
& =\lambda \sum_{n=-\infty}^{\infty} e^{-\pi \lambda\left(n+\frac{1}{2}\right)^{2}}\left\{\frac{2 \pi\left(n+\frac{1}{2}\right)}{z+n+\frac{1}{2}}\right\}\left(\frac{\sin \pi\left(z+n+\frac{1}{2}\right)}{\pi}\right)^{2} \\
& =\left(\frac{\cos \pi z}{\pi}\right)^{2} \sum_{n=-\infty}^{\infty} \frac{G_{\lambda}^{\prime}\left(n+\frac{1}{2}\right)}{\left(z-n-\frac{1}{2}\right)} .
\end{aligned}
$$


Now (1.17) follows from (5.2) and (5.3) by Fourier inversion. The proof of (1.18) is essentially the same. We note that (1.17) and (1.18) are both special cases of a general formula for Fourier transforms given in [35, Theorem 9].

\section{Proof of Theorems 5 and 6}

Let $\delta=2 N+2$, and let $p$ be a trigonometric polynomial of degree at most $N$. The Fourier expansions (1.5) and (3.4) imply that

$$
\begin{aligned}
\int_{\mathbb{R} / \mathbb{Z}}\left|\theta_{3}\left(x, i \lambda^{-1}\right)-p(x)\right| \mathrm{d} x & \geq\left|\int_{\mathbb{R} / \mathbb{Z}}\left\{\theta_{3}\left(x, i \lambda^{-1}\right)-p(x)\right\} \operatorname{sgn}(\cos \pi \delta x) \mathrm{d} x\right| \\
& =\left|\int_{\mathbb{R} / \mathbb{Z}} \theta_{3}\left(x, i \lambda^{-1}\right) \operatorname{sgn}(\cos \pi \delta x) \mathrm{d} x\right| \\
& =\left|\frac{2}{\pi} \sum_{n=-\infty}^{\infty} \frac{(-1)^{n}}{2 n+1} e^{-\pi \lambda^{-1}\left(n+\frac{1}{2}\right)^{2} \delta^{2}}\right| \\
& =\int_{-\frac{1}{2}}^{\frac{1}{2}} \theta_{1}\left(u, i \lambda^{-1} \delta^{2}\right) \mathrm{d} u,
\end{aligned}
$$

which proves (1.20). The representation of $\theta_{3}\left(v, i \lambda^{-1}\right)$ in (1.12), and the second representation of $k_{\lambda, N}$ in (1.19) imply that

$$
\theta_{3}\left(x, i \lambda^{-1}\right)-k_{\lambda, N}(x)=\lambda^{\frac{1}{2}} \sum_{m=-\infty}^{\infty}\left\{e^{-\pi \lambda(x+m)^{2}}-K_{\delta^{-2} \lambda}(\delta(x+m))\right\}
$$

for all $x \in \mathbb{R} / \mathbb{Z}$. The identity $G_{\delta^{-2} \lambda}(\delta x)=G_{\lambda}(x)$ and (3.2) give

$$
\operatorname{sgn}\left\{\theta_{3}\left(x, i \lambda^{-1}\right)-k_{\lambda, N}(x)\right\}=\operatorname{sgn}(\cos \pi \delta x) ;
$$

hence for $p=k_{\lambda, N}$ we have equality in (6.1). To prove uniqueness, note that in order to have equality in (6.1) we must have

$$
p\left(\frac{n+\frac{1}{2}}{\delta}\right)=\theta_{3}\left(\frac{n+\frac{1}{2}}{\delta}, i \lambda^{-1}\right)
$$

for $n=0,1,2, \ldots, 2 N+1$. Since the degree of $p(x)$ is at most $N$, such a polynomial exists and is unique [37. Vol II, page 1], and we showed in (6.2) that $k_{\lambda, N}$ already satisfies (6.3). This completes the proof of Theorem 5

We now prove the minorant part of Theorem [6] Let $\delta=N+1$. Let $q$ be a trigonometric polynomial of degree at most $N$ satisfying $q(x) \leq \theta_{3}\left(x, i \lambda^{-1}\right)$ at each $x \in \mathbb{R} \backslash \mathbb{Z}$. We use the fact that the degree of $q$ is at most $N$ (and $\delta=N+1$ ), and we apply (1.11) to obtain

$$
\begin{aligned}
\int_{\mathbb{R} / \mathbb{Z}} q(x) \mathrm{d} x & =\delta^{-1} \sum_{n=0}^{N} q\left(\frac{n+\frac{1}{2}}{\delta}\right) \\
& \leq \delta^{-1} \sum_{n=0}^{N} \theta_{3}\left(\frac{n+\frac{1}{2}}{\delta}, i \lambda^{-1}\right)=\theta_{2}\left(0, i \lambda^{-1} \delta^{2}\right) .
\end{aligned}
$$

This proves (1.25). Equations (1.12) and (1.21) imply that

$$
\theta_{3}\left(x, i \lambda^{-1}\right)-l_{\lambda, N}(x)=\lambda^{\frac{1}{2}} \sum_{m=-\infty}^{\infty}\left\{e^{-\pi \lambda(x+m)^{2}}-L_{\delta^{-2} \lambda}(\delta(x+m))\right\} \geq 0
$$


for all $x \in \mathbb{R} / \mathbb{Z}$, which proves the first inequality in (1.23). Since $L_{\lambda}$ interpolates the values of $G_{\lambda}$ at $\mathbb{Z}+\frac{1}{2}$, we have equality in (6.5), and hence

$$
l_{\lambda, N}\left(\frac{n+\frac{1}{2}}{\delta}\right)=\theta_{3}\left(\frac{n+\frac{1}{2}}{\delta}, i \lambda^{-1}\right)
$$

for $n=0,1, \ldots, N$. Moreover, (1.21) and (1.14) imply that

$$
\int_{\mathbb{R} / \mathbb{Z}} l_{\lambda, N}(x) \mathrm{d} x=\lambda^{\frac{1}{2}} \int_{-\infty}^{\infty} L_{\delta^{-2} \lambda}(\delta x) \mathrm{d} x=\theta_{2}\left(0, i \lambda^{-1} \delta^{2}\right) .
$$

Therefore equality occurs in (1.25) for $q=l_{\lambda, N}$. It remains to establish uniqueness. If equality occurs in (6.4), then

$$
q\left(\frac{n+\frac{1}{2}}{\delta}\right)=\theta_{3}\left(\frac{n+\frac{1}{2}}{\delta}, i \lambda^{-1}\right)=l_{\lambda, N}\left(\frac{n+\frac{1}{2}}{\delta}\right)
$$

for $n=0, \ldots, N$. Since $q$ and $l_{\lambda, N}$ both minorize $\theta_{3}\left(x, i \lambda^{-1}\right)$, their derivatives at the points $\delta^{-1}\left(n+\frac{1}{2}\right)$ where $n=0, \ldots, N$ must be equal. Hence $q$ satisfies $2 N+2$ conditions which determine a unique trigonometric polynomial of degree at most $N$ [37. Vol. II, p. 23], and therefore $q=l_{\lambda, N}$. The proof for the extremal majorizing trigonometric polynomial proceeds along analogous lines using interpolation points at $\delta^{-1} n$ for $n=0, \ldots, N$.

\section{PART II: Distribution theOREMS}

\section{The Paley-Wiener theorem for distributions}

Let $\mathcal{D}(\mathbb{R}) \subseteq \mathcal{S}(\mathbb{R}) \subseteq \mathcal{E}(\mathbb{R})$ be the usual spaces of $C^{\infty}$ functions on $\mathbb{R}$ as defined in the work of L. Schwartz [33], and let $\mathcal{E}^{\prime}(\mathbb{R}) \subseteq \mathcal{S}^{\prime}(\mathbb{R}) \subseteq \mathcal{D}^{\prime}(\mathbb{R})$ be the corresponding dual spaces of distributions. Our notation and terminology for distributions follows that of [13, and precise definitions for these spaces are given in [13, Section 2.3]. We write $\varphi(x)$ for a generic element in the space $\mathcal{S}(\mathbb{R})$ of Schwartz functions. If $T$ in $\mathcal{S}^{\prime}(\mathbb{R})$ is a tempered distribution we write $T(\varphi)$ for the value of $T$ at $\varphi$. Then the Fourier transform of $T$ is the tempered distribution $\widehat{T}$ defined by

$$
\widehat{T}(\varphi)=T(\widehat{\varphi}),
$$

where

$$
\widehat{\varphi}(y)=\int_{-\infty}^{\infty} \varphi(x) e(-y x) \mathrm{d} x
$$

is the Fourier transform of the function $\varphi$. Functions $g: \mathbb{R} \rightarrow \mathbb{R}$ in any $L^{p}$ class or with polynomial growth can be regarded as elements of $\mathcal{S}^{\prime}(\mathbb{R})$, and we will usually make the identification

$$
g(\varphi)=\int_{-\infty}^{\infty} g(x) \varphi(x) \mathrm{d} x
$$

for all $\varphi$ in $\mathcal{S}(\mathbb{R})$.

We recall the following form of the Paley-Wiener theorem for distributions, which is obtained by combining Theorem 1.7.5 and Theorem 1.7.7 in [18].

Theorem 14 (Paley-Wiener for distributions). Let $\delta>0$, and let $U$ be a tempered distribution in $\mathcal{S}^{\prime}(\mathbb{R})$ with Fourier transform $\widehat{U}$ supported in the compact interval $[-\delta, \delta]$. Then $\widehat{U}$ belongs to $\mathcal{E}^{\prime}(\mathbb{R})$, and

$$
z \mapsto F(z)=\widehat{U}_{\xi}(e(\xi z))
$$


defines an entire function of the complex variable $z=x+i y$ such that

$$
|F(z)| \ll_{B}(1+|z|)^{B} \exp \{2 \pi \delta|y|\}
$$

for some number $B \geq 0$ and all $z$ in $\mathbb{C}$. Moreover, the entire function $F(z)$ satisfies the identity

$$
U(\varphi)=\int_{-\infty}^{\infty} F(x) \varphi(x) d x
$$

for all $\varphi$ in $\mathcal{S}(\mathbb{R})$.

Conversely, suppose that $F(z)$ is an entire function of the complex variable $z$ that satisfies the inequality (7.1) for some numbers $B \geq 0$ and $\delta>0$. Then there exists a tempered distribution $V$ in $\mathcal{S}^{\prime}(\mathbb{R})$ such that $\widehat{V}$ belongs to $\mathcal{E}^{\prime}(\mathbb{R}), \widehat{V}$ is supported on the compact interval $[-\delta, \delta]$,

$$
F(z)=\widehat{V}_{\xi}(e(\xi z))
$$

and

$$
V(\varphi)=\int_{-\infty}^{\infty} F(x) \varphi(x) d x
$$

for all $\varphi$ in $\mathcal{S}(\mathbb{R})$.

Here we write $\widehat{U}_{\xi}$ to indicate that the distribution $\widehat{U}$ is acting on the function $\xi \mapsto(e(\xi z))$.

\section{Optimal integration}

Throughout Part II of this paper we let $\lambda$ denote a parameter on an interval $I \subset \mathbb{R}$. Then we consider a family of real valued even functions $x \mapsto G(\lambda, x)$ satisfying the following properties for each $\lambda \in I$.

(i) The function $x \mapsto G(\lambda, x)$ is continuous on $\mathbb{R}$ and differentiable on $\mathbb{R} /\{0\}$.

(ii) There exist constants $C=C(\lambda)>0$ and $\epsilon=\epsilon(\lambda)>0$ such that, for all $x \in \mathbb{R}$ and $t \in \mathbb{R}$,

$$
|G(\lambda, x)| \leq \frac{C}{(1+|x|)^{1+\epsilon}} \quad \text { and } \quad|\widehat{G}(\lambda, t)| \leq \frac{C}{(1+|t|)^{1+\epsilon}} .
$$

(iii) The Fourier transform $t \mapsto \widehat{G}(\lambda, t)$ is non-negative and radially non-increasing.

Depending on the type of problem to be treated (minorant, majorant or best approximation), we will require one additional hypothesis about the family $G(\lambda, x)$, for each $\lambda \in I$.

(iv) (Minorant) There is a unique extremal minorant $z \mapsto L(\lambda, z)$ of exponential type $2 \pi$ that interpolates the values of $G(\lambda, x)$ at $\mathbb{Z}+\frac{1}{2}$.

(v) (Majorant) There is a unique extremal majorant $z \mapsto M(\lambda, z)$ of exponential type $2 \pi$ that interpolates the values of $G(\lambda, x)$ at $\mathbb{Z}$.

(vi) (Best Approximation) There is a unique best approximation $z \mapsto K(\lambda, z)$ of exponential type $\pi$ that interpolates the values of $G(\lambda, x)$ at $\mathbb{Z}+\frac{1}{2}$ and satisfies

$$
\operatorname{sgn}(\cos \pi x)\{G(\lambda, x)-K(\lambda, x)\} \geq 0 .
$$


TABLE 1. Examples of solutions to the Beurling-Selberg problem.

\begin{tabular}{|c|c|c|c|}
\hline$G(\lambda, x)$ & Minorant & Majorant & Best Approximation \\
\hline$e^{-\pi \lambda x^{2}}$ & $\sum_{n \neq 0}(-1)^{n} \lambda^{-\frac{1}{2}} e^{-\pi \lambda^{-1} n^{2}}$ & $\sum_{n \neq 0} \lambda^{-\frac{1}{2}} e^{-\pi \lambda^{-1} n^{2}}$ & $\sum_{n=-\infty}^{\infty} \frac{(-1)^{n} \lambda^{-\frac{1}{2}} e^{-\pi \lambda^{-1}\left(n+\frac{1}{2}\right)^{2}}}{\pi\left(n+\frac{1}{2}\right)}$ \\
\hline$e^{-\lambda|x|}$ & $\frac{2}{\lambda}-\operatorname{csch}\left(\frac{\lambda}{2}\right)$ & $\operatorname{coth}\left(\frac{\lambda}{2}\right)-\frac{2}{\lambda}$ & $\frac{2}{\lambda}-\frac{2}{\lambda} \operatorname{sech}\left(\frac{\lambda}{2}\right)$ \\
\hline$\frac{2 \lambda}{\lambda^{2}+4 \pi^{2} x^{2}}$ & $\frac{2}{e^{\lambda}+1}$ & $\frac{2}{e^{\lambda}-1}$ & $\sum_{n=-\infty}^{\infty}(-1)^{n} \frac{e^{-\lambda\left|n+\frac{1}{2}\right|}}{\pi\left(n+\frac{1}{2}\right)}$ \\
\hline
\end{tabular}

We will call $\{x \mapsto G(\lambda, x)\}_{\lambda \in I}$ a minorant family if it satisfies properties (i)-(iv) above. The notions of majorant family and best approximation family are defined analogously using (v) and (vi) instead of (iv), respectively.

Observe that hypotheses (iv), (v) and (vi) do not need to coexist. Indeed, each problem can be treated independently. Examples of families of functions satisfying the conditions (i)-(iv) listed above that we have in mind for potential applications in this paper are given in Table 1 (note that in these cases $\lambda \in(0, \infty)$ ), together with the minimal values of the corresponding integrals. Our goal here is to be able to integrate the parameter $\lambda$ with respect to a suitable non-negative Borel measure on $I$ and obtain the solution to a different extremal problem. One might first guess that the class of suitable measures $\nu$ on $I$ would consist of those measures for which the function

$$
g(x)=\int_{I} G(\lambda, x) \mathrm{d} \nu(\lambda)
$$

is well defined, and that this would be the function to be approximated. Such a method was carried out in [3, [4] and [14]. It turns out that this condition is unnecessarily restrictive, and in order to find the optimal minimal conditions to be imposed on the measure $\nu$ one must look at things on the Fourier transform side.

We will illustrate what this condition should be in the minorant case. Define the difference function

$$
D(\lambda, x)=G(\lambda, x)-L(\lambda, x) \geq 0 .
$$

The minimal integral corresponds to

$$
\int_{-\infty}^{\infty}\{G(\lambda, x)-L(\lambda, x)\} \mathrm{d} x=\widehat{D}(\lambda, 0) .
$$

If we succeed in our attempt to integrate the parameter $\lambda$, we will end up solving an extremal problem for which the value of the minimal integral is given by (and thus we want to impose the finiteness)

$$
\int_{I} \int_{-\infty}^{\infty}\{G(\lambda, x)-L(\lambda, x)\} \mathrm{d} x \mathrm{~d} \nu(\lambda)=\int_{I} \widehat{D}(\lambda, 0) \mathrm{d} \nu(\lambda)<\infty .
$$

We will show that this is also a sufficient condition.

Suppose $\nu$ is a non-negative Borel measure on $I$ satisfying (8.1). Since

$$
|\widehat{D}(\lambda, t)| \leq \widehat{D}(\lambda, 0)
$$


for all $t \in \mathbb{R}$, we observe that the function

$$
t \mapsto \int_{I} \widehat{D}(\lambda, t) \mathrm{d} \nu(\lambda)
$$

is well defined. In particular, from the classical Paley-Wiener theorem, the Fourier transform $t \mapsto \widehat{L}(\lambda, t)$ is supported on $[-1,1]$, and therefore

$$
\int_{I} \widehat{D}(\lambda, t) \mathrm{d} \nu(\lambda)=\int_{I} \widehat{G}(\lambda, t) \mathrm{d} \nu(\lambda)
$$

for $|t| \geq 1$. We are now in a position to state the three main results of Part II of the paper. In the following theorems we write

$$
[\alpha, \beta]^{c}=(-\infty, \alpha) \cup(\beta, \infty)
$$

for the complement in $\mathbb{R}$ of a closed interval $[\alpha, \beta]$.

Theorem 15 (Distribution theorem - minorant). Let $\{x \mapsto G(\lambda, x)\}_{\lambda \in I}$ be a minorant family and $\nu$ be a non-negative Borel measure on I satisfying

$$
\int_{I} \int_{-\infty}^{\infty}\{G(\lambda, x)-L(\lambda, x)\} d x d \nu(\lambda)<\infty .
$$

Let $g: \mathbb{R} \rightarrow \mathbb{R}$ be a function on $\mathcal{S}^{\prime}(\mathbb{R})$ that is continuous on $\mathbb{R} /\{0\}$, differentiable on $\mathbb{R} /\{0\}$, and such that

$$
\widehat{g}(\varphi)=\int_{-\infty}^{\infty}\left\{\int_{I} \widehat{G}(\lambda, t) d \nu(\lambda)\right\} \varphi(t) d t
$$

for all Schwartz functions $\varphi$ supported on $[-1,1]^{c}$. Then there exists a unique extremal minorant $l(z)$ of exponential type $2 \pi$ for $g(x)$. The function $l(x)$ interpolates the values of $g(x)$ at $\mathbb{Z}+\frac{1}{2}$ and satisfies

$$
\int_{-\infty}^{\infty}\{g(x)-l(x)\} d x=\int_{I} \int_{-\infty}^{\infty}\{G(\lambda, x)-L(\lambda, x)\} d x d \nu(\lambda) .
$$

Theorem 16 (Distribution theorem - majorant). Let $\{x \mapsto G(\lambda, x)\}_{\lambda \in I}$ be a majorant family and $\nu$ be a non-negative Borel measure on I satisfying

$$
\int_{I} \int_{-\infty}^{\infty}\{M(\lambda, x)-G(\lambda, x)\} d x d \nu(\lambda)<\infty .
$$

Let $g: \mathbb{R} \rightarrow \mathbb{R}$ be a function on $\mathcal{S}^{\prime}(\mathbb{R})$ that is continuous on $\mathbb{R}$, differentiable on $\mathbb{R} /\{0\}$, and such that

$$
\widehat{g}(\varphi)=\int_{-\infty}^{\infty}\left\{\int_{I} \widehat{G}(\lambda, t) d \nu(\lambda)\right\} \varphi(t) d t
$$

for all Schwartz functions $\varphi$ supported on $[-1,1]^{c}$. Then there exists a unique extremal majorant $m(z)$ of exponential type $2 \pi$ for $g(x)$. The function $m(x)$ interpolates the values of $g(x)$ at $\mathbb{Z}$ and satisfies

$$
\int_{-\infty}^{\infty}\{m(x)-g(x)\} d x=\int_{I} \int_{-\infty}^{\infty}\{M(\lambda, x)-G(\lambda, x)\} d x d \nu(\lambda) .
$$

Theorem 17 (Distribution theorem - best approximation). Let $\{x \mapsto G(\lambda, x)\}_{\lambda \in I}$ be a best approximation family and $\nu$ be a non-negative Borel measure on I satisfying

$$
\int_{I} \int_{-\infty}^{\infty}|G(\lambda, x)-K(\lambda, x)| d x d \nu(\lambda)<\infty .
$$


Let $g: \mathbb{R} \rightarrow \mathbb{R}$ be a function on $\mathcal{S}^{\prime}(\mathbb{R})$ that is continuous on $\mathbb{R} /\{0\}$, and such that

$$
\widehat{g}(\varphi)=\int_{-\infty}^{\infty}\left\{\int_{I} \widehat{G}(\lambda, t) d \nu(\lambda)\right\} \varphi(t) d t
$$

for all Schwartz functions $\varphi$ supported on $\left[-\frac{1}{2}, \frac{1}{2}\right]^{c}$. Then there exists a unique best approximation $k(z)$ of exponential type $\pi$ for $g(x)$. The function $k(x)$ interpolates the values of $g(x)$ at $\mathbb{Z}+\frac{1}{2}$, satisfying

$$
\operatorname{sgn}(\cos \pi x)\{g(x)-k(x)\} \geq 0
$$

and

$$
\int_{-\infty}^{\infty}|g(x)-k(x)| d x=\int_{I} \int_{-\infty}^{\infty}|G(\lambda, x)-K(\lambda, x)| d x d \nu(\lambda) .
$$

Similar results can be stated for the problem of majorizing or minorizing by functions of exponential type $2 \pi \delta$, or determining best approximations by functions of type $\pi \delta$. It is a matter of changing the interpolation points to $\delta \mathbb{Z}$ or $\delta\left(\mathbb{Z}+\frac{1}{2}\right)$, and changing the support intervals to $[-\delta, \delta]^{c}$ in the case of minorants/majorants and to $\left[-\frac{\delta}{2}, \frac{\delta}{2}\right]^{c}$ in the case of best approximations. For simplicity, we will proceed in our exposition only with type $2 \pi$ for minorants/majorants and type $\pi$ for the best approximation problem.

The condition

$$
\widehat{g}(\varphi)=\int_{-\infty}^{\infty}\left\{\int_{I} \widehat{G}(\lambda, t) \mathrm{d} \nu(\lambda)\right\} \varphi(t) \mathrm{d} t
$$

for all Schwartz functions $\varphi$ supported on $[-\delta, \delta]^{c}$, that appears on the statements of the theorems, asserts that the Fourier transform $\widehat{g}$, which is a tempered distribution, is actually given by a function

$$
t \mapsto \int_{I} \widehat{G}(\lambda, t) \mathrm{d} \nu(\lambda)
$$

outside the interval $[-\delta, \delta]$. This is typical behavior of functions with polynomial growth that might have a Fourier transform given by a singular part supported on the origin plus an additional component given by a function outside the origin (e.g. the Fourier transform of $-\log |x|$ is given by $(2|t|)^{-1}$ away from the origin). It is clear in this context that the only information relevant for the Beurling-Selberg extremal problem is knowledge of the Fourier transform of the original function outside a compact interval.

Finally, we shall see that this method applied to the Gaussian family

$$
G(\lambda, x)=G_{\lambda}(x)=e^{-\pi \lambda x^{2}}
$$

is quite powerful, producing most of the previously known examples in the literature, as well as a wide class of new ones. In particular, we will be able to deal with families of functions such as

$$
\widetilde{G}(\alpha, x)=\log \left(\frac{x^{2}+\alpha^{2}}{x^{2}+4}\right) \quad \text { and } \quad \widetilde{G}(\alpha, x)=|x|^{\alpha} .
$$

We could also integrate the new parameter $\alpha$ to produce further examples. Although these families do not satisfy the original requirements (i)-(iv), this is a perfectly reasonable argument, since by Fubini's theorem an integral with respect to the parameter $\alpha$ will only produce a different measure $\nu$ for the original integration on the parameter $\lambda$ for the Gaussian. Therefore, there is no loss of generality 
in starting the procedure with a family of functions satisfying the regularity requirements (i)-(iv) and iterating the method as desired.

\section{Proofs of Theorems 15 and 16}

Here we give a detailed proof of Theorem 15. The proof of Theorem 16 follows the same general method.

First we construct the extreme minorant. Recall that

$$
D(\lambda, x)=G(\lambda, x)-L(\lambda, x) \geq 0 .
$$

Then for each $x \in \mathbb{R}$ we define the non-negative valued function

$$
d(x)=\int_{I} D(\lambda, x) \mathrm{d} \nu(\lambda) .
$$

It may happen that the value of $d(x)$ is $\infty$ at some points $x$. However, the function $x \mapsto d(x)$ is integrable on $\mathbb{R}$ because

$$
\int_{-\infty}^{\infty} d(x) \mathrm{d} x=\int_{I} \int_{-\infty}^{\infty} D(\lambda, x) \mathrm{d} x \mathrm{~d} \nu(\lambda)=\int_{I} \widehat{D}(\lambda, 0) \mathrm{d} \nu(\lambda)<\infty
$$

by the hypotheses of our theorem. Hence the Fourier transform $\widehat{d}(t)$ is a continuous function given by

$$
\begin{aligned}
\widehat{d}(t)=\int_{-\infty}^{\infty} d(x) e(-t x) \mathrm{d} x=\int_{-\infty}^{\infty} \int_{I} D(\lambda, x) e(-t x) \mathrm{d} \nu(\lambda) \mathrm{d} x \\
=\int_{I} \int_{-\infty}^{\infty} D(\lambda, x) e(-t x) \mathrm{d} x \mathrm{~d} \nu(\lambda)=\int_{I} \widehat{D}(\lambda, t) \mathrm{d} \nu(\lambda),
\end{aligned}
$$

and for $|t| \geq 1$ we have

$$
\widehat{d}(t)=\int_{I} \widehat{G}(\lambda, t) \mathrm{d} \nu(\lambda) .
$$

Let $U \in \mathcal{S}^{\prime}(\mathbb{R})$ be the tempered distribution determined by

$$
U(\varphi)=\int_{-\infty}^{\infty}\{g(x)-d(x)\} \varphi(x) \mathrm{d} x .
$$

We shall prove that the Fourier transform $\widehat{U}$ is supported on $[-1,1]$. In fact, for any $\varphi \in \mathcal{S}(\mathbb{R})$ with support in $[-1,1]^{c}$ we have

$$
\begin{aligned}
\widehat{U}(\varphi) & =\widehat{g}(\varphi)-\widehat{d}(\varphi) \\
& =\int_{-\infty}^{\infty}\left\{\int_{I} \widehat{G}(\lambda, t) \mathrm{d} \nu(\lambda)\right\} \varphi(t) \mathrm{d} t-\int_{-\infty}^{\infty} \widehat{d}(t) \varphi(t) \mathrm{d} t=0
\end{aligned}
$$

by (9.3) and the hypotheses of the theorem. By the Paley-Wiener theorem for distributions we find that $\widehat{U} \in \mathcal{E}^{\prime}(\mathbb{R})$, and therefore

$$
z \mapsto l(z)=\widehat{U}_{\xi}(e(\xi z))
$$

defines an entire function of exponential type $2 \pi$ such that

$$
U(\varphi)=\int_{-\infty}^{\infty} l(x) \varphi(x) \mathrm{d} x
$$

for all $\varphi \in \mathcal{S}(\mathbb{R})$. From (9.4) and (9.5) we conclude that

$$
d(x)=g(x)-l(x) \geq 0
$$


for almost all $x \in \mathbb{R}$. In particular, we get

$$
\begin{aligned}
\int_{-\infty}^{\infty}\{g(x)-l(x)\} \mathrm{d} x & =\int_{-\infty}^{\infty} d(x) \mathrm{d} x=\int_{I} \widehat{D}(\lambda, 0) \mathrm{d} \nu(\lambda) \\
& =\int_{I} \int_{-\infty}^{\infty}\{G(\lambda, x)-L(\lambda, x)\} \mathrm{d} x \mathrm{~d} \nu(\lambda)<\infty .
\end{aligned}
$$

Next we consider the interpolation points. Because of conditions (i) and (ii), the Poisson summation formula can be applied to $D(\lambda, x)$, giving a pointwise identity

$$
\sum_{n=-\infty}^{\infty} D(\lambda, x+n)=\sum_{k=-\infty}^{\infty} \widehat{D}(\lambda, k) e(x k) .
$$

From condition (iv) of our hypotheses we have $D\left(\lambda, n+\frac{1}{2}\right)=0$ for all $n \in \mathbb{Z}$. Therefore we apply (9.7) at $x=\frac{1}{2}$, and use the classical Paley-Wiener theorem. In this way we arrive at the identity

$$
\widehat{D}(\lambda, 0)=-\sum_{\substack{k=-\infty \\ k \neq 0}}^{\infty}(-1)^{k} \widehat{G}(\lambda, k) .
$$

Now we define the function

$$
d_{1}(x)=g(x)-l(x)
$$

We note that $d_{1}(x)$ is a non-negative, continuous function on $\mathbb{R} /\{0\}$ that is equal almost everywhere to $d(x)$ defined in (9.1), and thus in $L^{1}(\mathbb{R})$. Define a periodic function $p: \mathbb{R} / \mathbb{Z} \rightarrow \mathbb{R}^{+} \cup\{\infty\}$ by

$$
p(x)=\sum_{n \in \mathbb{Z}} d_{1}(n+x) .
$$

An application of Fubini's theorem provides

$$
\int_{\mathbb{R} / \mathbb{Z}} p(x) \mathrm{d} x=\int_{-\infty}^{\infty} d_{1}(x) \mathrm{d} x<\infty
$$

and therefore $p(x) \in L^{1}(\mathbb{R} / \mathbb{Z})$. Moreover, the Fourier coefficients of $p(x)$ satisfy

$$
\widehat{p}(k)=\widehat{d}_{1}(k)=\widehat{d}(k)
$$

for all $k \in \mathbb{Z}$. Convolution with the smoothing Féjer kernel

$$
F_{N}(x)=\frac{1}{N+1}\left(\frac{\sin \pi(N+1) x}{\sin \pi x}\right)^{2}
$$


produces the pointwise identity

$$
\begin{aligned}
p * F_{N}(x) & =\sum_{k=-N}^{N}\left(1-\frac{|k|}{N}\right) \widehat{p}(k) e(x k) \\
& =\widehat{d}(0)+\sum_{\substack{k=-N \\
k \neq 0}}^{N}\left(1-\frac{|k|}{N}\right) \widehat{d}(k) e(x k) \\
& =\widehat{d}(0)+\sum_{\substack{k=-N \\
k \neq 0}}^{N}\left(1-\frac{|k|}{N}\right) \int_{I} \widehat{G}(\lambda, k) \mathrm{d} \nu(\lambda) e(x k) \\
& =\widehat{d}(0)+\int_{I}\left\{\sum_{\substack{k=-N \\
k \neq 0}}^{N}\left(1-\frac{|k|}{N}\right) \widehat{G}(\lambda, k) e(x k)\right\} \mathrm{d} \nu(\lambda),
\end{aligned}
$$

where we have used (9.3). In particular, at $x=\frac{1}{2}$ we obtain

$$
\widehat{d}(0)=p * F_{N}\left(\frac{1}{2}\right)+\int_{I}\left\{\sum_{\substack{k=-N \\ k \neq 0}}^{N}(-1)^{k+1}\left(1-\frac{|k|}{N}\right) \widehat{G}(\lambda, k)\right\} \mathrm{d} \nu(\lambda) .
$$

By condition (iii) of the hypotheses, the integrand in (9.9) is non-negative. Moreover, by condition (ii) it converges absolutely to (9.8) as $N \rightarrow \infty$. Therefore, an application of Fatou's lemma together with (9.2) gives us

$$
\begin{aligned}
\widehat{d}(0) \geq \liminf _{N \rightarrow \infty} p * F_{N}\left(\frac{1}{2}\right) & +\liminf _{N \rightarrow \infty} \int_{I}\left\{\sum_{\substack{k=-N \\
k \neq 0}}^{N}(-1)^{k+1}\left(1-\frac{|k|}{N}\right) \widehat{G}(\lambda, k)\right\} \mathrm{d} \nu(\lambda) \\
\geq \liminf _{N \rightarrow \infty} p * F_{N}\left(\frac{1}{2}\right) & +\int_{I} \liminf _{N \rightarrow \infty}\left\{\sum_{\substack{k=-N \\
k \neq 0}}^{N}(-1)^{k+1}\left(1-\frac{|k|}{N}\right) \widehat{G}(\lambda, k)\right\} \mathrm{d} \nu(\lambda) \\
= & \liminf _{N \rightarrow \infty} p * F_{N}\left(\frac{1}{2}\right)+\int_{I} \widehat{D}(\lambda, 0) \mathrm{d} \nu(\lambda) \\
= & \liminf _{N \rightarrow \infty} p * F_{N}\left(\frac{1}{2}\right)+\widehat{d}(0),
\end{aligned}
$$

and since $p * F_{N}(x)$ is non-negative we conclude that

$$
\liminf _{N \rightarrow \infty} p * F_{N}\left(\frac{1}{2}\right)=0
$$


We now use the definition of $p(x)$, Fubini's theorem and Fatou's lemma again to arrive at

$$
\begin{aligned}
0=\liminf _{N \rightarrow \infty} & p * F_{N}\left(\frac{1}{2}\right)=\liminf _{N \rightarrow \infty} \int_{0}^{1} p(y) F_{N}\left(\frac{1}{2}-y\right) \mathrm{d} y \\
= & \liminf _{N \rightarrow \infty} \int_{0}^{1}\left\{\sum_{n \in \mathbb{Z}} d_{1}(n+y)\right\} F_{N}\left(\frac{1}{2}-y\right) \mathrm{d} y \\
= & \liminf _{N \rightarrow \infty} \sum_{n \in \mathbb{Z}}\left\{\int_{0}^{1} d_{1}(n+y) F_{N}\left(\frac{1}{2}-y\right) \mathrm{d} y\right\} \\
\geq & \sum_{n \in \mathbb{Z}} \liminf _{N \rightarrow \infty} \int_{0}^{1} d_{1}(n+y) F_{N}\left(\frac{1}{2}-y\right) \mathrm{d} y \\
& =\sum_{n \in \mathbb{Z}} d_{1}\left(n+\frac{1}{2}\right)
\end{aligned}
$$

where the last equality follows from the fact that $d_{1}(x)$ is continuous at the points $n+\frac{1}{2}, n \in \mathbb{Z}$. From (9.10) and the non-negativity of $d_{1}(x)$ we arrive at the implication

$$
d_{1}\left(n+\frac{1}{2}\right)=0 \Rightarrow g\left(n+\frac{1}{2}\right)=l\left(n+\frac{1}{2}\right)
$$

for all $n \in \mathbb{Z}$. From (9.6) and the fact that $g(x)$ is differentiable on $\mathbb{R} /\{0\}$ (by hypothesis) we also have

$$
g^{\prime}\left(n+\frac{1}{2}\right)=l^{\prime}\left(n+\frac{1}{2}\right)
$$

for all $n \in \mathbb{Z}$.

Finally, we show that the integral is minimal and we establish uniqueness. Assume that $\widetilde{l}(z)$ is a real entire function of exponential type $2 \pi$ such that

$$
\widetilde{l}(x) \leq g(x)
$$

for all $x \in \mathbb{R}$, and suppose that $\{g(x)-\widetilde{l}(x)\}$ is integrable. In this case the function

$$
j(z)=l(z)-\widetilde{l}(z)
$$

has exponential type $2 \pi$ and is integrable on $\mathbb{R}$. An application of [14, Lemma 4] together with (9.11) and (9.12) gives us

$$
\begin{aligned}
\widehat{j}(0) & =\lim _{N \rightarrow \infty} \sum_{n=-N}^{N}\left(1-\frac{|n|}{N}\right) j\left(n+\frac{1}{2}\right) \\
& =\lim _{N \rightarrow \infty} \sum_{n=-N}^{N}\left(1-\frac{|n|}{N}\right)\left(g\left(n+\frac{1}{2}\right)-\widetilde{l}\left(n+\frac{1}{2}\right)\right) \geq 0 .
\end{aligned}
$$

This plainly verifies that

$$
\int_{-\infty}^{\infty}\{g(x)-\widetilde{l}(x)\} \mathrm{d} x \geq \int_{-\infty}^{\infty}\{g(x)-l(x)\} \mathrm{d} x
$$

and establishes the minimality of the integral. If equality occurs in (9.13) we must have

$$
\widetilde{l}\left(n+\frac{1}{2}\right)=g\left(n+\frac{1}{2}\right)=l\left(n+\frac{1}{2}\right)
$$


for all $n \in \mathbb{Z}$. From (9.12) we also have

$$
\tilde{l}^{\prime}\left(n+\frac{1}{2}\right)=g^{\prime}\left(n+\frac{1}{2}\right)=l^{\prime}\left(n+\frac{1}{2}\right)
$$

for all $n \in \mathbb{Z}$. The interpolation conditions (9.14) and (9.15) imply that

$$
j\left(n+\frac{1}{2}\right)=j^{\prime}\left(n+\frac{1}{2}\right)=0
$$

for all $n \in \mathbb{Z}$. By a second application of [14, Lemma 4], we conclude that the entire function $j(z)$ is identically zero. This proves the uniqueness of the extremal minorant $l(z)$, and completes the proof.

In the proof of uniqueness in the majorant case, we will obtain

$$
j^{\prime}(n)=0
$$

for all $n \neq 0$, since the original function $g(x)$ is not assumed to be differentiable at the origin. A further application of [14, Lemma 4] shows that $j^{\prime}(0)=0$, and so leads to uniqueness.

\section{Proof of Theorem 17}

The approach here is similar to the proof of Theorem 16. We start by considering the difference function

$$
D(\lambda, x)=G(\lambda, x)-K(\lambda, x),
$$

and for each $x \in \mathbb{R}$ define the function

$$
d(x)=\int_{I} D(\lambda, x) \mathrm{d} \nu(\lambda) .
$$

From condition (vi) in the hypotheses, we know that

$$
\operatorname{sgn}(\cos \pi x) d(x) \geq 0,
$$

with this value possibly being infinity at some points. Observe, however, that $d(x)$ is integrable on $\mathbb{R}$, with

$$
\begin{aligned}
\int_{-\infty}^{\infty}|d(x)| \mathrm{d} x & =\int_{-\infty}^{\infty} \int_{I}|D(\lambda, x)| \mathrm{d} \nu(\lambda) \mathrm{d} x \\
& =\int_{I} \int_{-\infty}^{\infty}|G(\lambda, x)-K(\lambda, x)| \mathrm{d} x \mathrm{~d} \nu(\lambda)<\infty .
\end{aligned}
$$

An application of Fubini's theorem gives us

$$
\widehat{d}(t)=\int_{I} \widehat{D}(\lambda, t) \mathrm{d} \nu(\lambda)
$$

for all $t \in \mathbb{R}$. Because $z \mapsto K(\lambda, z)$ has exponential type $\pi$, we have

$$
\widehat{d}(t)=\int_{I} \widehat{G}(\lambda, t) \mathrm{d} \nu(\lambda)
$$

for $|t| \geq \frac{1}{2}$. Let $V \in \mathcal{S}^{\prime}(\mathbb{R})$ be the tempered distribution given by

$$
V(\varphi)=\int_{-\infty}^{\infty}\{g(x)-d(x)\} \varphi(x) \mathrm{d} x .
$$


We shall prove that the Fourier transform $\widehat{V}$ is supported on $\left[-\frac{1}{2}, \frac{1}{2}\right]$. In fact, for any $\varphi \in \mathcal{S}(\mathbb{R})$ with support in $\left[-\frac{1}{2}, \frac{1}{2}\right]^{c}$ we have

$$
\begin{aligned}
\widehat{V}(\varphi) & =\widehat{g}(\varphi)-\widehat{d}(\varphi) \\
& =\int_{-\infty}^{\infty}\left\{\int_{I} \widehat{G}(\lambda, t) \mathrm{d} \nu(\lambda)\right\} \varphi(t) \mathrm{d} t-\int_{-\infty}^{\infty} \widehat{d}(t) \varphi(t) \mathrm{d} t=0,
\end{aligned}
$$

by (10.2) and the hypotheses of the theorem. By the Paley-Wiener theorem for distributions we find out that $\widehat{V} \in \mathcal{E}^{\prime}(\mathbb{R})$ and

$$
z \mapsto k(z)=\widehat{V}_{\xi}(e(\xi z))
$$

defines an entire function of exponential type $\pi$ such that

$$
V(\varphi)=\int_{-\infty}^{\infty} k(x) \varphi(x) \mathrm{d} x
$$

for all $\varphi \in \mathcal{S}(\mathbb{R})$. From (10.3) and (10.4) we conclude that

$$
d(x)=g(x)-k(x)
$$

for almost all $x \in \mathbb{R}$. In particular,

$$
\begin{aligned}
\int_{-\infty}^{\infty}|g(x)-k(x)| \mathrm{d} x & =\int_{-\infty}^{\infty}|d(x)| \mathrm{d} x \\
& =\int_{I} \int_{-\infty}^{\infty}|G(\lambda, x)-K(\lambda, x)| \mathrm{d} x \mathrm{~d} \nu(\lambda)<\infty .
\end{aligned}
$$

Since $g(x)$ is continuous on $\mathbb{R} /\{0\}$ (by hypothesis) and $k(x)$ is the restriction to $\mathbb{R}$ of an entire function, expressions (10.1) and (10.5) imply that

$$
\operatorname{sgn}(\cos \pi x)\{g(x)-k(x)\} \geq 0
$$

for all $x \in \mathbb{R}$. In particular, we must have

$$
g\left(n+\frac{1}{2}\right)=k\left(n+\frac{1}{2}\right)
$$

for all $n \in \mathbb{Z}$.

Recall that the function $x \mapsto \operatorname{sgn}(\cos \pi x)$ is periodic on $\mathbb{R}$ with period 2 and has the Fourier series expansion

$$
\operatorname{sgn}(\cos \pi x)=\lim _{N \rightarrow \infty} \frac{1}{\pi} \sum_{n=-N-1}^{N} \frac{(-1)^{n}}{n+\frac{1}{2}} e\left(\left(n+\frac{1}{2}\right) x\right) .
$$

Moreover, the partial sums on the right of (10.6) are uniformly bounded. If $h(z)$ is an entire function of exponential type $\pi$ that is integrable on $\mathbb{R}$, then its Fourier transform $\widehat{h}(t)$ will be supported on $\left[-\frac{1}{2}, \frac{1}{2}\right]$. Then we will have

$$
\begin{aligned}
\int_{-\infty}^{\infty} \operatorname{sgn}(\cos \pi x) & h(x) \mathrm{d} x \\
& =\lim _{N \rightarrow \infty} \frac{1}{\pi} \sum_{n=-N-1}^{N} \frac{(-1)^{n}}{n+\frac{1}{2}} \int_{-\infty}^{\infty} h(x) e\left(\left(n+\frac{1}{2}\right) x\right) \mathrm{d} x \\
& =\lim _{N \rightarrow \infty} \frac{1}{\pi} \sum_{n=-N-1}^{N} \frac{(-1)^{n}}{n+\frac{1}{2}} \widehat{h}\left(-n-\frac{1}{2}\right)=0 .
\end{aligned}
$$


Now assume that $\widetilde{k}(z)$ is an entire function of exponential type $\pi$ such that

$$
\int_{-\infty}^{\infty}|g(x)-\widetilde{k}(x)| \mathrm{d} x<\infty .
$$

In this case, the function $\{k(x)-\widetilde{k}(x)\}$ has exponential type $\pi$ and is integrable on $\mathbb{R}$. Thus, using (10.7) we obtain

$$
\begin{aligned}
\int_{-\infty}^{\infty}|g(x)-\widetilde{k}(x)| \mathrm{d} x \geq\left|\int_{-\infty}^{\infty} \operatorname{sgn}(\cos \pi x)\{g(x)-\widetilde{k}(x)\} \mathrm{d} x\right| \\
=\left|\int_{-\infty}^{\infty} \operatorname{sgn}(\cos \pi x)\{(g(x)-k(x))+(k(x)-\widetilde{k}(x))\} \mathrm{d} x\right| \\
=\left|\int_{-\infty}^{\infty} \operatorname{sgn}(\cos \pi x)\{g(x)-k(x)\} \mathrm{d} x\right| \\
=\int_{-\infty}^{\infty}|g(x)-k(x)| \mathrm{d} x
\end{aligned}
$$

proving the minimality of the integral. If equality occurs in (10.8) we must have

$$
\widetilde{k}\left(n+\frac{1}{2}\right)=g\left(n+\frac{1}{2}\right)=k\left(n+\frac{1}{2}\right)
$$

for all $n \in \mathbb{Z}$. Therefore

$$
z \mapsto k(z)-\widetilde{k}(z)
$$

is an entire function of exponential type at most $\pi$ and takes the value zero at each point of the set $\mathbb{Z}+\frac{1}{2}$. From basic interpolation theorems for entire functions of exponential type (see [37, Vol. II, p. 275]), we conclude that the entire function (10.9) is identically zero, proving the uniqueness. This completes the proof.

\section{PART III: APPLICATIONS}

By combining the results from Part I and Part II, we are able to solve the Beurling-Selberg extremal problem for a wide class of even functions, extending the results in [3], 4], 14] and 22]. As mentioned in the Introduction, some of the $L^{1}(\mathbb{R})$ approximations (without the one-sided conditions) recover results of Sz.-Nagy [26], 32. Sz.-Nagy's results are applicable to functions with a Fourier transform that satisfy certain monotonicity conditions for $t \geq \delta>0$ and are either even or odd. It is an interesting open problem to determine if the extremals for all such functions can be obtained from our methods. Throughout Part III of this paper we consider extremal minorants/majorants of exponential type $2 \pi$, and best approximations of exponential type $\pi$, unless otherwise specified.

\section{Positive Definite FunCtions}

Recall that in Part I we worked with the family of Gaussian functions

$$
G_{\lambda}(x)=e^{-\pi \lambda x^{2}},
$$

where $\lambda>0$ is a parameter. The Fourier transform $t \mapsto \widehat{G}_{\lambda}(t)$ is given by

$$
\widehat{G}_{\lambda}(t)=\lambda^{-\frac{1}{2}} e^{-\pi \lambda^{-1} t^{2}} .
$$

In Theorems 1, 2, and 3, we constructed, for each $\lambda>0$, the extremal minorant $L_{\lambda}(z)$, the extremal majorant $M_{\lambda}(z)$, and the best approximation $K_{\lambda}(z)$, for $G_{\lambda}(x)$. 
These functions satisfy all the hypotheses (i)-(vi) of the distribution theorems. The values of the minimal integrals are given by

$$
\begin{aligned}
& \int_{-\infty}^{\infty}\left\{G_{\lambda}(x)\right.\left.-L_{\lambda}(x)\right\} \mathrm{d} x \\
&= \lambda^{-\frac{1}{2}}\left(1-\theta_{2}\left(0, i \lambda^{-1}\right)\right)=\sum_{\substack{n=-\infty \\
n \neq 0}}^{\infty}(-1)^{n+1} \widehat{G}_{\lambda}(n), \\
& \int_{-\infty}^{\infty}\left\{M_{\lambda}(x)-G_{\lambda}(x)\right\} \mathrm{d} x \\
&=\lambda^{-\frac{1}{2}}\left(\theta_{3}\left(0, i \lambda^{-1}\right)-1\right)=\sum_{n=-\infty}^{\infty} \widehat{G}_{\lambda}(n), \\
& \int_{-\infty}^{\infty}\left|G_{\lambda}(x)-K_{\lambda}(x)\right| \mathrm{d} x \\
&=\lambda^{-\frac{1}{2}} \int_{-\frac{1}{2}}^{\frac{1}{2}} \theta_{1}\left(u, i \lambda^{-1}\right) \mathrm{d} u=\frac{1}{\pi} \sum_{n=-\infty}^{\infty} \frac{(-1)^{n}}{n+\frac{1}{2}} \widehat{G}_{\lambda}\left(n+\frac{1}{2}\right) .
\end{aligned}
$$

From the three expressions above and the transformation formulas (1.10), (1.11) and (1.12) we obtain the estimates

$$
\begin{aligned}
\int_{-\infty}^{\infty}\left\{G_{\lambda}(x)-L_{\lambda}(x)\right\} \mathrm{d} x & =\left\{\begin{array}{l}
O\left(\lambda^{-\frac{1}{2}} e^{-\pi \lambda^{-1}}\right) \text { as } \lambda \rightarrow 0, \\
O\left(\lambda^{-\frac{1}{2}}\right) \text { as } \lambda \rightarrow \infty,
\end{array}\right. \\
\int_{-\infty}^{\infty}\left\{M_{\lambda}(x)-G_{\lambda}(x)\right\} \mathrm{d} x & =\left\{\begin{array}{l}
O\left(\lambda^{-\frac{1}{2}} e^{-\pi \lambda^{-1}}\right) \text { as } \lambda \rightarrow 0, \\
O(1) \text { as } \lambda \rightarrow \infty,
\end{array}\right. \\
\int_{-\infty}^{\infty}\left|G_{\lambda}(x)-K_{\lambda}(x)\right| \mathrm{d} x & =\left\{\begin{array}{l}
O\left(\lambda^{-\frac{1}{2}} e^{-\pi(4 \lambda)^{-1}}\right) \text { as } \lambda \rightarrow 0, \\
O\left(\lambda^{-\frac{1}{2}}\right) \text { as } \lambda \rightarrow \infty
\end{array}\right.
\end{aligned}
$$

In order to apply Theorems 15, 16, and 17 to the Gaussian family, we require a non-negative measure $\nu$ defined on the Borel subsets of $I=(0, \infty)$. We further require that integrals with respect to $\nu$ over the parameter $\lambda$ appearing in (11.1), (11.2) and (11.3) are finite. The estimates (11.4), (11.5) and (11.6) show that this class of measures is relatively large because of the very fast decay at the origin. One should compare this class of measures with the ones used in [3], [4, and [14] to fully notice the improvement.

As a first application we present the following result.

Corollary 18. Let $\nu$ be a finite non-negative Borel measure on $(0, \infty)$ and consider the function $g: \mathbb{R} \rightarrow \mathbb{R}$ given by

$$
g(x)=\int_{0}^{\infty} e^{-\pi \lambda x^{2}} d \nu(\lambda)
$$

(i) There exists a unique extremal minorant $l(z)$ of exponential type $2 \pi$ for $g(x)$. The function $l(x)$ interpolates the values of $g(x)$ at $\mathbb{Z}+\frac{1}{2}$ and satisfies

$$
\int_{-\infty}^{\infty}\{g(x)-l(x)\} d x=\int_{0}^{\infty}\left\{\sum_{\substack{n=-\infty \\ n \neq 0}}^{\infty}(-1)^{n+1} \widehat{G}_{\lambda}(n)\right\} d \nu(\lambda) .
$$


(ii) There exists a unique extremal majorant $m(z)$ of exponential type $2 \pi$ for $g(x)$. The function $m(x)$ interpolates the values of $g(x)$ at $\mathbb{Z}$ and satisfies

$$
\int_{-\infty}^{\infty}\{m(x)-g(x)\} d x=\int_{0}^{\infty}\left\{\sum_{\substack{n=-\infty \\ n \neq 0}}^{\infty} \widehat{G}_{\lambda}(n)\right\} d \nu(\lambda) .
$$

(iii) There exists a unique best approximation $k(z)$ of exponential type $\pi$ for $g(x)$. The function $k(x)$ interpolates the values of $g(x)$ at $\mathbb{Z}+\frac{1}{2}$ and satisfies

$$
\int_{-\infty}^{\infty}|g(x)-k(x)| d x=\int_{0}^{\infty}\left\{\frac{1}{\pi} \sum_{n=-\infty}^{\infty} \frac{(-1)^{n}}{n+\frac{1}{2}} \widehat{G}_{\lambda}\left(n+\frac{1}{2}\right)\right\} d \nu(\lambda) .
$$

Due to a classical result of Schoenberg (see [27, Theorems 2 and 3]), a function $g: \mathbb{R} \rightarrow \mathbb{R}$ admits the representation (11.7) if and only if its radial extension to $\mathbb{R}^{N}$ is positive definite, for all $N \in \mathbb{N}$, or equivalently if the function $g\left(|x|^{1 / 2}\right)$ is completely monotone. Recall that a function $f(t)$ is completely monotone for $t \geq 0$ if

$$
(-1)^{n} f^{(n)}(t) \geq 0 \text { for } 0<t<\infty, \text { and } n=1,2,3, \ldots,
$$

and

$$
f(0)=f(0+)
$$

The last condition expresses the continuity of $f(t)$ at the origin. Using this characterization we arrive at the following interesting examples contemplated by our Corollary 18.

Example 1. $g(x)=e^{-\alpha|x|^{2 r}}, \quad \alpha>0$, and $0<r \leq 1$.

Example 2. $g(x)=\left(x^{2}+\alpha^{2}\right)^{-\beta}, \alpha>0$ and $\beta>0$.

The first example shows that we can recover all the theory for the exponential function $g(x)=e^{-\lambda|x|}$ developed in [3, 4] and [14, from the family of Gaussian functions and the distribution theorems. The second example includes the Poisson kernel $g(x)=2 \lambda /\left(\lambda^{2}+4 \pi^{2} x^{2}\right), \lambda>0$. The values of the minimal integrals in these cases are collected in Table 1 of Section 8 .

Recently, Chandee and Soundararajan 6 used the extremal functions for $f(x)=$ $\log \left(x^{2} /\left(x^{2}+4\right)\right)$, described in [3, to obtain improved upper bounds for $\left|\zeta\left(\frac{1}{2}+i t\right)\right|$, assuming the Riemann Hypothesis (RH). They remarked that the extremal functions for $f(x)=\log \left(\left(x^{2}+\alpha^{2}\right) /\left(x^{2}+4\right)\right)$, where $\alpha \neq 0$, which were not contemplated in the previous literature, arise in bounding $\left|\zeta\left(\frac{1}{2} \pm \alpha+i t\right)\right|$ assuming RH. They note that knowledge of the associated extremal functions would lead to improved results in the critical strip for estimates of the type

$$
\log \zeta(s)=O\left\{\frac{(\log t)^{1-2 \alpha}}{\log \log t}\right\},
$$

where $s=(1 / 2+\alpha)+i t$ and $0 \leq \alpha<1 / 2$. Inequality (11.8) can be found in 34, Theorem 14.5].

Here we are able to obtain the extremal functions for this class as an application of Corollary 18

Example 3. $g(x)=-\log \left(\frac{x^{2}+\alpha^{2}}{x^{2}+\beta^{2}}\right)$, for $0<\alpha<\beta$. 
Indeed, for $0<\alpha<\beta$ consider the non-negative finite measure

$$
\mathrm{d} \nu(\lambda)=\frac{\left\{e^{-\pi \lambda \alpha^{2}}-e^{-\pi \lambda \beta^{2}}\right\}}{\lambda} \mathrm{d} \lambda
$$

and observe that

$$
-\log \left(\frac{x^{2}+\alpha^{2}}{x^{2}+\beta^{2}}\right)=\int_{0}^{\infty} e^{-\pi \lambda x^{2}} \frac{\left\{e^{-\pi \lambda \alpha^{2}}-e^{-\pi \lambda \beta^{2}}\right\}}{\lambda} \mathrm{d} \lambda .
$$

In particular, the values of the minimal integrals in the one-sided approximations are given by

$$
\int_{-\infty}^{\infty}\left\{-\log \left(\frac{x^{2}+\alpha^{2}}{x^{2}+\beta^{2}}\right)-l_{\alpha, \beta}(x)\right\} \mathrm{d} x=2 \log \left(\frac{1+e^{-2 \pi \alpha}}{1+e^{-2 \pi \beta}}\right)
$$

and

$$
\int_{-\infty}^{\infty}\left\{m_{\alpha, \beta}(x)+\log \left(\frac{x^{2}+\alpha^{2}}{x^{2}+\beta^{2}}\right)\right\} \mathrm{d} x=2 \log \left(\frac{1-e^{-2 \pi \beta}}{1-e^{-2 \pi \alpha}}\right) .
$$

12. Extremal Functions FOR $|x|^{\sigma}$

In this section we write $s=\sigma+i t$ for a complex variable, and we define the meromorphic function $s \mapsto \gamma(s)$ by

$$
\gamma(s)=\pi^{-s / 2} \Gamma\left(\frac{s}{2}\right) .
$$

The function $\gamma(s)$ is analytic on $\mathbb{C}$ except for simple poles at the points $s=$ $0,-2,-4, \ldots$ It also occurs in the functional equation

$$
\gamma(s) \zeta(s)=\gamma(1-s) \zeta(1-s),
$$

where $\zeta(s)$ is the Riemann zeta-function.

Lemma 19. Let $0<\delta$ and let $\varphi(t)$ be a Schwartz function supported on $[-\delta, \delta]^{c}$. Then

$$
s \mapsto \int_{-\infty}^{\infty}|t|^{-s-1} \varphi(t) d t
$$

defines an entire function of $s$, and the identity

$$
\gamma(s+1) \int_{-\infty}^{\infty}|t|^{-s-1} \varphi(t) d t=\gamma(-s) \int_{-\infty}^{\infty}|x|^{s} \widehat{\varphi}(x) d x
$$

holds in the half plane $\{s \in \mathbb{C}:-1<\sigma\}$. In particular, the function on the right of (12.3) is analytic at the points $s=0,2,4, \ldots$.

Proof. Because $\varphi(t)$ is supported in $[-\delta, \delta]^{c}$, the function $t \mapsto|t|^{-s-1} \varphi(t)$ is integrable on $\mathbb{R}$ for all complex values $s$. Hence by Morera's theorem the integral on the right of (12.2) defines an entire function. The identity (12.3) holds in the infinite strip $\{s \in \mathbb{C}:-1<\sigma<0\}$ by [30, Lemma 1, p. 117], and therefore it holds in the half plane $\{s \in \mathbb{C}:-1<\sigma\}$ by analytic continuation. The left hand side of (12.3) is clearly analytic at each point of $\{s \in \mathbb{C}:-1<\sigma\}$, hence the right hand side of (12.3) is also analytic at each point of this half plane. 
Lemma 19 asserts that, for $-1<\sigma$ and $\sigma \neq 0,2,4, \ldots$, the Fourier transform of the function $x \mapsto \gamma(-\sigma)|x|^{\sigma}$ is given by the function

$$
t \mapsto \gamma(\sigma+1)|t|^{-\sigma-1}
$$

outside the interval $[-\delta, \delta]$.

We intend to apply the distribution theorems with the Gaussian. Toward this end, consider the non-negative Borel measure $\nu_{\sigma}$ on $(0, \infty)$ given by

$$
\mathrm{d} \nu_{\sigma}(\lambda)=\lambda^{-\frac{\sigma}{2}-1} \mathrm{~d} \lambda
$$

and observe that we have

$$
\int_{0}^{\infty} \widehat{G}_{\lambda}(t) \mathrm{d} \nu_{\sigma}(\lambda)=\gamma(\sigma+1)|t|^{-\sigma-1}
$$

For $-1<\sigma$, the measure $\nu_{\sigma}$ is admissible for the minorant and best approximation problems according to the asymptotics (11.4) and (11.6). For the majorant problem we shall require that $0<\sigma$, according to the asymptotics (11.5).

It will be convenient to introduce the Dirichlet $L$-function $L(s, \chi)$, where $\chi$ is the unique non-principal Dirichlet character to the modulus 4 . This $L$-function is defined in the half plane $\{s \in \mathbb{C}: 1<\sigma\}$ by the absolutely convergent series

$$
L(s, \chi)=\sum_{n=1}^{\infty} \chi(n) n^{-s}=\sum_{n=0}^{\infty}(-1)^{n}(2 n+1)^{-s} .
$$

Then the $L$-function extends by analytic continuation to an entire function of $s$. As $\chi$ is a primitive character, the $L$-function satisfies the functional equation

$$
\xi(s, \chi)=\xi(1-s, \chi),
$$

where $s \mapsto \xi(s, \chi)$ is the entire function defined by

$$
\xi(s, \chi)=\left(\frac{4}{\pi}\right)^{\frac{s+1}{2}} \Gamma\left(\frac{s+1}{2}\right) L(s, \chi) .
$$

Lemma 20. Let $\sigma>-1$. Then we have

$$
\int_{-\infty}^{\infty} \int_{0}^{\infty}\left|G_{\lambda}(x)-K_{\lambda}(x)\right| \lambda^{-\frac{\sigma}{2}-1} d \lambda d x=\left(\frac{4}{\pi}\right)^{\frac{3+\sigma}{2}} \Gamma\left(\frac{1+\sigma}{2}\right) L(2+\sigma, \chi) .
$$

Proof. Using Fubini's theorem, (1.5), and (1.13), we get

$$
\begin{aligned}
\int_{-\infty}^{\infty} \int_{0}^{\infty} \mid G_{\lambda}(x)- & K_{\lambda}(x) \mid \lambda^{-\frac{\sigma}{2}-1} \mathrm{~d} \lambda \mathrm{d} x \\
& =\int_{0}^{\infty}\left\{\lambda^{-\frac{1}{2}} \int_{-\frac{1}{2}}^{\frac{1}{2}} \theta_{1}\left(u, i \lambda^{-1}\right) \mathrm{d} u\right\} \lambda^{-\frac{\sigma}{2}-1} \mathrm{~d} \lambda \\
& =\int_{0}^{\infty}\left\{\sum_{n=-\infty}^{\infty} \frac{(-1)^{n}}{\pi\left(n+\frac{1}{2}\right)} e^{-\pi \lambda^{-1}\left(n+\frac{1}{2}\right)^{2}}\right\} \lambda^{\frac{-\sigma-3}{2}} \mathrm{~d} \lambda
\end{aligned}
$$


Because

$$
\begin{aligned}
\int_{0}^{\infty}\left\{\sum_{n=-\infty}^{\infty} \frac{1}{\pi\left|n+\frac{1}{2}\right|} e^{-\pi \lambda^{-1}\left(n+\frac{1}{2}\right)^{2}}\right\} \lambda^{\frac{-\sigma-3}{2}} \mathrm{~d} \lambda \\
=\sum_{n=-\infty}^{\infty} \frac{1}{\pi\left|n+\frac{1}{2}\right|}\left\{\int_{0}^{\infty} \lambda^{\frac{-\sigma-3}{2}} e^{-\pi \lambda^{-1}\left(n+\frac{1}{2}\right)^{2}} \mathrm{~d} \lambda\right\} \\
=\gamma(1+\sigma) \sum_{n=-\infty}^{\infty} \frac{1}{\pi}\left|n+\frac{1}{2}\right|^{-\sigma-2}<\infty,
\end{aligned}
$$

the partial sums of the series on the right of (12.7) are dominated by an integrable function. Thus we have

$$
\begin{aligned}
\int_{0}^{\infty}\left\{\sum_{n=-\infty}^{\infty} \frac{(-1)^{n}}{\pi\left(n+\frac{1}{2}\right)} e^{-\pi \lambda^{-1}\left(n+\frac{1}{2}\right)^{2}}\right\} \lambda^{\frac{-\sigma-3}{2}} \mathrm{~d} \lambda \\
=\sum_{n=-\infty}^{\infty} \frac{(-1)^{n}}{\pi\left(n+\frac{1}{2}\right)}\left\{\int_{0}^{\infty} \lambda^{\frac{-\sigma-3}{2}} e^{-\pi \lambda^{-1}\left(n+\frac{1}{2}\right)^{2}} \mathrm{~d} \lambda\right\} \\
=\gamma(1+\sigma) \sum_{n=-\infty}^{\infty} \frac{(-1)^{n}}{\pi\left(n+\frac{1}{2}\right)}\left|n+\frac{1}{2}\right|^{-\sigma-1} \\
=\left(\frac{4}{\pi}\right)^{\frac{3+\sigma}{2}} \Gamma\left(\frac{1+\sigma}{2}\right) L(2+\sigma, \chi) .
\end{aligned}
$$

Identities (12.7) and (12.8) imply that the identity (12.6) holds for $\sigma>-1$.

The following lemma can be proved in a similar manner using Theorems 2 and 3. and then applying termwise integration to the series (1.6) and (1.7).

Lemma 21. Let $\sigma>-1$. Then we have

$$
\int_{-\infty}^{\infty} \int_{0}^{\infty}\left\{G_{\lambda}(x)-L_{\lambda}(x)\right\} \lambda^{-\frac{\sigma}{2}-1} d \lambda d x=\left(2-2^{1-\sigma}\right) \gamma(1+\sigma) \zeta(1+\sigma) .
$$

Let $\sigma>0$. Then we have

$$
\int_{-\infty}^{\infty} \int_{0}^{\infty}\left\{M_{\lambda}(x)-G_{\lambda}(x)\right\} \lambda^{-\frac{\sigma}{2}-1} d \lambda d x=2 \gamma(1+\sigma) \zeta(1+\sigma) .
$$

Theorems 15, 16 and 17 now apply. The values of the integrals in the following corollary are obtained from Lemmas 20 and 21.

Corollary 22. Let $-1<\sigma$ with $\sigma \neq 0,2,4, \ldots$, and let

$$
g_{\sigma}(x)=\gamma(-\sigma)|x|^{\sigma} .
$$

(i) There exists a unique extremal minorant $l_{\sigma}(z)$ of exponential type $2 \pi$ for $g_{\sigma}(x)$. The function $l_{\sigma}(x)$ interpolates the values of $g_{\sigma}(x)$ at $\mathbb{Z}+\frac{1}{2}$ and satisfies

$$
\int_{-\infty}^{\infty}\left\{g_{\sigma}(x)-l_{\sigma}(x)\right\} d x=\left(2-2^{1-\sigma}\right) \gamma(1+\sigma) \zeta(1+\sigma) .
$$


(ii) If $0<\sigma$, there exists a unique extremal majorant $m_{\sigma}(z)$ of exponential type $2 \pi$ for $g_{\sigma}(x)$. The function $m_{\sigma}(x)$ interpolates the values of $g_{\sigma}(x)$ at $\mathbb{Z}$ and satisfies

$$
\int_{-\infty}^{\infty}\left\{m_{\sigma}(x)-g_{\sigma}(x)\right\} d x=2 \gamma(1+\sigma) \zeta(1+\sigma) .
$$

(iii) There exists a unique best approximation $k_{\sigma}(z)$ of exponential type $\pi$ for $g_{\sigma}(x)$. The function $k_{\sigma}(x)$ interpolates the values of $g_{\sigma}(x)$ at $\mathbb{Z}+\frac{1}{2}$, satisfying

$$
\operatorname{sgn}(\cos \pi x)\left\{g_{\sigma}(x)-k_{\sigma}(x)\right\} \geq 0
$$

and

$$
\int_{-\infty}^{\infty}\left|g_{\sigma}(x)-k_{\sigma}(x)\right| d x=\left(\frac{4}{\pi}\right)^{\frac{3+\sigma}{2}} \Gamma\left(\frac{1+\sigma}{2}\right) L(2+\sigma, \chi) .
$$

Parts (i) and (ii) of Corollary 22 provide a complete description of the extreme minorants and extreme majorants associated to $x \mapsto|x|^{\sigma}$. For $\sigma \leq-1$ these functions are not integrable at the origin, and therefore no extremals exist, and for $\sigma=2 k, k \in \mathbb{Z}^{+}$, these functions are entire, have only polynomial growth, and therefore the extremal problem is trivial. Previous results had been obtained in [3] and [4] for the functions $x \mapsto|x|^{\sigma},-1<\sigma<1$, and in 22] for the functions $x \mapsto|x|^{2 k+1}$, with $k \in \mathbb{Z}^{+}$. We note that the best approximation identified in part (iii) of Corollary 22 was already obtained by Ganzburg [9] (see also [11, Example $3.2])$.

Next we consider Hilbert-type inequalities. It is well known that there is a simple relationship between the solution of the Beurling-Selberg extremal problem for a function $g: \mathbb{R} \rightarrow \mathbb{R}$ and the existence of optimal bounds for Hermitian forms involving the Fourier transform $\widehat{g}$. Such bounds for Hermitian forms are called Hilbert-type inequalities. Detailed proofs of these inequalities can be found in 35 , Theorem 16] or [3, Theorem 7.1]. In particular, here we report on the Hilberttype inequalities that follow from Corollary 22. They involve the same kernel as the classical discrete Hardy-Littlewood-Sobolev inequality (see [16, p. 288]), and generalize the result contained in [3, Corollary 7.2].

Corollary 23. Let $\xi_{1}, \xi_{2}, \ldots, \xi_{N}$ be real numbers such that $0<\delta \leq\left|\xi_{m}-\xi_{n}\right|$ whenever $m \neq n$. Let $a_{1}, a_{2}, \ldots, a_{N}$ be complex numbers. If $0<\sigma<1$, then

$$
-\frac{\left(2-2^{2-\sigma}\right) \zeta(\sigma)}{\delta^{\sigma}} \sum_{n=1}^{N}\left|a_{n}\right|^{2} \leq \sum_{m=1}^{N} \sum_{\substack{n=1 \\ n \neq m}}^{N} \frac{a_{m} \bar{a}_{n}}{\left|\xi_{m}-\xi_{n}\right|^{\sigma}} .
$$

If $\sigma=1$, then

$$
-\frac{\log 4}{\delta} \sum_{n=1}^{N}\left|a_{n}\right|^{2} \leq \sum_{m=1}^{N} \sum_{\substack{n=1 \\ n \neq m}}^{N} \frac{a_{m} \bar{a}_{n}}{\left|\xi_{m}-\xi_{n}\right|}
$$

and if $1<\sigma$, then

$$
-\frac{\left(2-2^{2-\sigma}\right) \zeta(\sigma)}{\delta^{\sigma}} \sum_{n=1}^{N}\left|a_{n}\right|^{2} \leq \sum_{m=1}^{N} \sum_{\substack{n=1 \\ n \neq m}}^{N} \frac{a_{m} \bar{a}_{n}}{\left|\xi_{m}-\xi_{n}\right|^{\sigma}} \leq \frac{2 \zeta(\sigma)}{\delta^{\sigma}} \sum_{n=1}^{N}\left|a_{n}\right|^{2} .
$$

The constants appearing in these inequalities are the best possible. 


\section{FURTHER EXAMPLES}

We complete our list of applications with two additional examples that follow from the distribution theorems.

Corollary 24. Let $\alpha \geq 0$ and consider

$$
x \mapsto \tau_{\alpha}(x)=-\log \left(x^{2}+\alpha^{2}\right) .
$$

(i) There exists a unique extremal minorant $l_{\alpha}$ of exponential type $2 \pi$ for $\tau_{\alpha}$. The function $l_{\alpha}$ interpolates the values of $\tau_{\alpha}$ at $\mathbb{Z}+\frac{1}{2}$, and satisfies

$$
\int_{-\infty}^{\infty}\left\{\tau_{\alpha}(x)-l_{\alpha}(x)\right\} d x=2 \log \left(1+e^{-2 \pi \alpha}\right)
$$

(ii) If $0<\alpha$, there exists a unique extremal majorant $m_{\alpha}$ of exponential type $2 \pi$ for $\tau_{\alpha}$. The function $m_{\alpha}$ interpolates the values of $\tau_{\alpha}$ at $\mathbb{Z}$, and satisfies

$$
\int_{-\infty}^{\infty}\left\{m_{\alpha}(x)-\tau_{\alpha}(x)\right\} d x=-2 \log \left(1-e^{-2 \pi \alpha}\right) .
$$

(iii) There exists a unique best approximation $k_{\alpha}$ of exponential type $\pi$ for $\tau_{\alpha}$. The function $k_{\alpha}$ interpolates the values of $\tau_{\alpha}$ at $\mathbb{Z}+\frac{1}{2}$, and satisfies

$$
\operatorname{sgn}(\cos \pi x)\left\{\tau_{\alpha}(x)-k_{\alpha}(x)\right\} \geq 0
$$

and

$$
\int_{-\infty}^{\infty}\left|\tau_{\alpha}(x)-k_{\alpha}(x)\right| d x=\int_{0}^{\infty}\left\{\frac{1}{\pi} \sum_{n=-\infty}^{\infty} \frac{(-1)^{n}}{n+\frac{1}{2}} \widehat{G}_{\lambda}\left(n+\frac{1}{2}\right)\right\} \frac{e^{-\pi \lambda \alpha^{2}}}{\lambda} d \lambda .
$$

Proof. For $0 \leq \alpha$ we have the identity

$$
-\log \left(x^{2}+\alpha^{2}\right)=\int_{0}^{\infty} \frac{\left\{e^{-\pi \lambda\left(x^{2}+\alpha^{2}\right)}-e^{-\pi \lambda}\right\}}{\lambda} \mathrm{d} \lambda .
$$

Let $\varphi$ be a Schwartz function supported in $[-\delta, \delta]^{c}$. An application of Fubini's theorem leads to the identity

$$
\begin{aligned}
& \int_{-\infty}^{\infty}-\log \left(x^{2}+\alpha^{2}\right) \widehat{\varphi}(x) \mathrm{d} x \\
&=\int_{-\infty}^{\infty}\left\{\int_{0}^{\infty} \frac{\left\{e^{-\pi \lambda\left(x^{2}+\alpha^{2}\right)}-e^{-\pi \lambda}\right\}}{\lambda} \mathrm{d} \lambda\right\} \widehat{\varphi}(x) \mathrm{d} x \\
&=\int_{0}^{\infty} \int_{-\infty}^{\infty} \frac{\left\{e^{-\pi \lambda\left(x^{2}+\alpha^{2}\right)}-e^{-\pi \lambda}\right\}}{\lambda} \widehat{\varphi}(x) \mathrm{d} x \mathrm{~d} \lambda \\
&=\int_{0}^{\infty}\left\{\int_{-\infty}^{\infty} \widehat{G}_{\lambda}(t) \varphi(t) \mathrm{d} t\right\} \frac{e^{-\pi \lambda \alpha^{2}}}{\lambda} \mathrm{d} \lambda \\
&=\int_{-\infty}^{\infty}\left\{\int_{0}^{\infty} \widehat{G}_{\lambda}(t) \frac{e^{-\pi \lambda \alpha^{2}}}{\lambda} \mathrm{d} \lambda\right\} \varphi(t) \mathrm{d} t .
\end{aligned}
$$


Equation (13.2) provides the Fourier transform of $-\log \left(x^{2}+\alpha^{2}\right)$ outside a compact interval $[-\delta, \delta]$. We can therefore apply the distribution theorems (Theorems 15 , [16 and 17) with the Gaussian family, and measure $\nu$ on $I=(0, \infty)$ given by

$$
\mathrm{d} \nu(\lambda)=\frac{e^{-\pi \lambda \alpha^{2}}}{\lambda} \mathrm{d} \lambda .
$$

According to the asymptotics (11.4), (11.5) and (11.6), if $\alpha>0$ we can treat the three approximation problems, and if $\alpha=0$ we can only treat the minorant and the best approximation problem (which is in agreement with the fact that $-\log |x|$ is unbounded from above). The special case of $-\log |x|$ (when $\alpha=0$ ) was obtained in [3] and [4].

Corollary 25. Let $n$ be a non-negative integer and define $h_{n}$ by

$$
h_{n}(x)=(-1)^{n+1} x^{2 n} \log \left(x^{2}\right) .
$$

(i) There exists a unique extremal minorant $l_{n}$ of exponential type $2 \pi$ for $h_{n}$. The function $l_{n}$ interpolates the values of $h_{n}$ at $\mathbb{Z}+\frac{1}{2}$ and satisfies

$$
\int_{-\infty}^{\infty}\left\{h_{n}(x)-l_{n}(x)\right\} d x=\left(2-2^{1-2 n}\right)(2 n) !(2 \pi)^{-2 n} \zeta(2 n+1) .
$$

(ii) If $n>0$, there exists a unique extremal majorant $m_{n}$ of exponential type $2 \pi$ for $h_{n}$. The function $m_{n}$ interpolates the values of $h_{n}$ at $\mathbb{Z}$, and satisfies

$$
\int_{-\infty}^{\infty}\left\{m_{n}(x)-h_{n}(x)\right\} d x=2(2 n) !(2 \pi)^{-2 n} \zeta(2 n+1) .
$$

(iii) There exists a unique best approximation $k_{n}$ of exponential type $\pi$ for $h_{n}$. The function $k_{n}$ interpolates the values of $h_{n}$ at $\mathbb{Z}+\frac{1}{2}$, and satisfies

$$
\operatorname{sgn}(\cos \pi x)\left\{h_{n}(x)-k_{n}(x)\right\} \geq 0
$$

and

$$
\int_{-\infty}^{\infty}\left|h_{n}(x)-k_{n}(x)\right| d x=\frac{2}{\pi}(2 n) !(2 \pi)^{-2 n} L(2 n+2, \chi) .
$$

Proof. Let $\varphi$ be a Schwartz function supported in $[-\delta, \delta]^{c}$. We make use of identity (13.1) (with $\alpha=0$ ) and repeated applications of Fubini's theorem. We find that

$$
\begin{aligned}
\int_{-\infty}^{\infty} h_{n}(x) \widehat{\varphi}(x) \mathrm{d} x & =\int_{-\infty}^{\infty}(-1)^{n} x^{2 n}\left\{\int_{0}^{\infty} \frac{\left\{e^{-\pi \lambda x^{2}}-e^{-\pi \lambda}\right\}}{\lambda} \mathrm{d} \lambda\right\} \widehat{\varphi}(x) \mathrm{d} x \\
& =\int_{0}^{\infty} \int_{-\infty}^{\infty}(-1)^{n} x^{2 n} \frac{\left\{e^{-\pi \lambda x^{2}}-e^{-\pi \lambda}\right\}}{\lambda} \widehat{\varphi}(x) \mathrm{d} x \mathrm{~d} \lambda \\
& =\frac{1}{(2 \pi)^{2 n}} \int_{0}^{\infty}\left\{\int_{-\infty}^{\infty} \widehat{G}_{\lambda}^{(2 n)}(t) \varphi(t) \mathrm{d} t\right\} \frac{1}{\lambda} \mathrm{d} \lambda \\
& =\frac{1}{(2 \pi)^{2 n}} \int_{-\infty}^{\infty}\left\{\int_{0}^{\infty} \frac{\widehat{G}_{\lambda}^{(2 n)}(t)}{\lambda} \mathrm{d} \lambda\right\} \varphi(t) \mathrm{d} t \\
& =\frac{1}{(2 \pi)^{2 n}} \int_{-\infty}^{\infty}\left(\frac{\mathrm{d}}{\mathrm{d} t}\right)^{2 n}\left\{\int_{0}^{\infty} \frac{\widehat{G}_{\lambda}(t)}{\lambda} \mathrm{d} \lambda\right\} \varphi(t) \mathrm{d} t
\end{aligned}
$$


Then using (12.4), the last integral on the right of (13.3) can be written as

$$
\begin{aligned}
& =\frac{\gamma(1)}{(2 \pi)^{2 n}} \int_{-\infty}^{\infty}\left(\frac{\mathrm{d}}{\mathrm{d} t}\right)^{2 n}|t|^{-1} \varphi(t) \mathrm{d} t \\
& =\frac{(2 n) !}{(2 \pi)^{2 n}} \int_{-\infty}^{\infty}|t|^{-2 n-1} \varphi(t) \mathrm{d} t .
\end{aligned}
$$

Hence, the Fourier transform of $h_{n}$ in the distribution sense, outside the compact interval $[-\delta, \delta]$, is given by the function

$$
t \mapsto \frac{(2 n) !}{(2 \pi)^{2 n}}|t|^{-2 n-1}=\frac{(2 n) !}{(2 \pi)^{2 n}} \gamma(2 n+1)^{-1} \int_{0}^{\infty} \widehat{G}_{\lambda}(t) \mathrm{d} \nu_{2 n}(\lambda),
$$

where the last identity follows from (12.4) with $\sigma=2 n$. An application of Theorems 15. 16 and 17 with measure

$$
\mathrm{d} \nu(\lambda)=\frac{(2 n) !}{(2 \pi)^{2 n}} \gamma(2 n+1)^{-1} \lambda^{-n-1} \mathrm{~d} \lambda,
$$

together with the formulas in Lemmas 20 and 21, lead to the desired result.

\section{ACKNOWLEDGments}

The research of the first author was supported by the National Science Foundation under agreement DMS-0635607. The research of the third author was supported by the National Science Foundation under agreement DMS-0603282. The first author would also like to acknowledge support from the Capes/Fulbright grant BEX 1710-04-4 and the Homer Lindsey Bruce Fellowship from the University of Texas. The authors are thankful to K. Soundararajan and E. Bombieri for their enlightening comments during the preparation of this work. The authors also wish to thank the referee for calling their attention to work of M. I. Ganzburg [9, [10, 11] and M. G. Krein [19.

\section{REFERENCES}

[1] J. T. Barton, H. L. Montgomery, and J. D. Vaaler, Note on a Diophantine inequality in several variables, Proc. Amer. Math. Soc. 129 (2000), 337-345. MR.1800228 (2002j:11090)

[2] E. Carneiro, Sharp approximations to the Bernoulli periodic functions by trigonometric polynomials, J. Approx. Theory 154 (2008), 90-104. MR2474766 (2009k:42002)

[3] E. Carneiro and J. D. Vaaler, Some extremal functions in Fourier analysis, II, Trans. Amer. Math. Soc., 362 (2010), 5803-5843. MR2661497 (2011g:41013)

[4] E. Carneiro and J. D. Vaaler, Some extremal functions in Fourier analysis, III, Constr. Approx., 31 No. 2 (2010), 259-288. MR.2581230(2011a:41034)

[5] V. Chandee and E. Carneiro, Bounding $\zeta(s)$ in the critical strip, J. Number Theory, 131 (2011), 363-384. MR2739041 (2011k:11109)

[6] V. Chandee and K. Soundararajan, Bounding $\left|\zeta\left(\frac{1}{2}+i t\right)\right|$ on the Riemann hypothesis, Bull. Lond. Math. Soc. 43, No. 2 (2011), 243-250. MR2781205

[7] K. Chandrasekharan, Elliptic Functions, Springer-Verlag, Berlin, 1985. MR808396 (87e:11058)

[8] T. Ganelius, On one-sided approximation by trigonometric polynomials, Math. Scan. 4 (1956), 247-258. MR0088596(19:545d) 
[9] M. I. Ganzburg, Criteria for best approximation of locally integrable functions in $L(\mathbb{R})$, Current problems of Summation and Approximation of Functions and their Applications, Dnepropetrovsk Gos. University, Dnepropetrovsk, 1983, 11-16 (Russian). MR.750116 (85h:41065)

[10] M. I. Ganzburg, Limit theorems and best constants of approximation theory, Handbook on Analytic-Computational Methods in Applied Mathematics, ed. G. A. Anastassiou, CRC Press, Boca Raton, 2000, 507-569. MR.1769932 (2001f:41032)

[11] M. I. Ganzburg, L-approximation to non-periodic functions, Journal of Concrete and Applicable Mathematics, 8, No. 2 (2010), 208-215. MR2606258 (2011b:41041)

[12] M. I. Ganzburg and D. S. Lubinsky, Best approximating entire functions to $|x|^{\alpha}$ in $L^{2}$, Complex analysis and dynamical systems III, 93-107, Contemp. Math. 455, Amer. Math. Soc., Providence, RI, 2008. MR2408163(2010h:41016)

[13] L. Grafakos, Classical and Modern Fourier Analysis, Pearson, Upper Saddle River, New Jersey, 2004. MR2449250

[14] S. W. Graham and J. D. Vaaler, A class of extremal functions for the Fourier transform, Tran. Amer. Math. Soc. 265 (1981), 283-382. MR607121 (82i:42008)

[15] S. W. Graham and J. D. Vaaler, Extremal functions for the Fourier transform and the large sieve, Topics in Classical Number Theory, Vol. I, II (Budapest, 1981), Colloq. Math. Soc. Janós Bolyai, 34, North-Holland, Amsterdam, 599-615. MR781154 (87a:11087)

[16] G. H. Hardy, G. Polya, and J. E. Littlewod, Inequalities, Cambridge University Press, 1988. MR.944909

[17] J. Holt and J. D. Vaaler, The Beurling-Selberg extremal functions for a ball in the Euclidean space, Duke Mathematical Journal 83 (1996), 203-247. MR.1388849 (97f:30038)

[18] L. Hörmander, Linear Partial Differential Operators, Springer-Verlag, 1969. MR.0248435

[19] M. G. Krein, On the best approximation of continuously differentiable functions on the whole real axis, Dokl. Akad. Nauk SSSR, 18 (1938), 615-624 (Russian).

[20] X. J. Li and J. D. Vaaler, Some trigonometric extremal functions and the Erdös-Turán type inequalities, Indiana Univ. Math. J. 48 (1999), no. 1, 183-236. MR1722198(2001a:11136)

[21] F. Littmann, One-sided approximation by entire functions, J. Approx. Theory 141 (2006), no. 1, 1-7. MR2246686 (2007c:41016)

[22] F. Littmann, Entire majorants via Euler-Maclaurin summation, Trans. Amer. Math. Soc. 358 (2006), no. 7, 2821-2836. MR2216247 (2007f:42002)

[23] F. Littmann, Entire approximations to the truncated powers, Constr. Approx. 22 (2005), no. 2, 273-295. MR2148534(2006e:41009)

[24] H. L. Montgomery, The analytic principle of the large sieve, Bull. Amer. Math. Soc. 84 (1978), no. 4, 547-567. MR0466048 (57:5931)

[25] H. L. Montgomery and R. C. Vaughan, Hilbert's inequality, J. London Math. Soc. (2) 8 (1974), 73-81. MR0337775(49:2544)

[26] B. Sz.-Nagy, Über gewisse Extremalfragen bei transformierten trigonometrischen Entwicklungen II, Ber. Math.-Phys. Kl. Sächs. Akad. Wiss. Leipzig 91 (1939).

[27] I. J. Schoenberg, Metric spaces and completely monotone functions, Ann. of Math. (2) 39 (1938), 811-841. MR1503439

[28] A. Selberg, Remarks on sieves, Proc. 1972 Number Theory conf. (Univ. of Colorado, Boulder), pp 205-216; also in Atle Selberg: Collected Papers, Vol. I, Springer-Verlag, Berlin, 1989, pp. 609-615. MR0389802 (52:10632)

[29] A. Selberg, Lectures on Sieves, Atle Selberg: Collected Papers, Vol. II, Springer-Verlag, Berlin, 1991, pp. 65-247. MR1295844 (95g:01032)

[30] E. Stein, Singular Integrals and Differentiability Properties of Functions, Princeton University Press, 1970. MR0290095 (44:7280)

[31] E. Stein and G. Weiss, Introduction to Fourier Analysis on Euclidean Spaces, Princeton University Press, 1971. MR0304972(46:4102)

[32] H. Shapiro, Topics in Approximation Theory, Lecture Notes in Mathematics 187, SpringerVerlag, 1971. MR0437981 (55:10902)

[33] L. Schwartz, Théorie des Distributions, vols. I et II, Hermann 1950, 1951. MR0209834 $(35: 730)$

[34] E.C. Titchmarsh, The Theory of the Riemann Zeta-function, 2nd edition, Clarendon Press, Oxford, 1986. MR882550 (88c:11049)

[35] J. D. Vaaler, Some extremal functions in Fourier analysis, Bull. Amer. Math. Soc. 12 (1985), 183-215. MR776471 (86g:42005) 
[36] J. D. Vaaler, Refinements of the Erdös-Turán inequality, Number Theory with an emphasis on the Markoff spectrum (Provo, UT, 1991), Lecture Notes in Mathematics, no. 147, Dekker, 1993, 263-269. MR1219340(94c:11069)

[37] A. Zygmund, Trigonometric Series, Cambridge University Press, 1959. MR0107776 (21:6498)

impa-Instituto de Matematica Pura e Aplicada-Estrada Dona Castorina, 110, Rio DE JANEIRo, 22460-320, BrAZIL

E-mail address: carneiro@impa.br

Department of Mathematics, North Dakota State University, Fargo, North Dakota 58105-5075

E-mail address: friedrich.littmann@ndsu.edu

Department of Mathematics, University of Texas at Austin, Austin, Texas 78712-1082

E-mail address: vaaler@math.utexas.edu 